\title{
Evidências no desenvolvimento de habilidades socioemocionais via tecnologias educacionais digitais/analógicas para crianças do século XXI: um mapeamento sistemático do estado da arte como fomento a gestores para apoio à políticas públicas brasileiras
}

\author{
Title: Evidence on the development of socioemotional skills via digital educational \\ technologies for children of the 21st century: a systematic mapping of the state of the art as \\ an incentive for managers to support Brazilian public policies
}

Felipe Faustino de Souza Universidade Federal de Sergipe (UFS)- PROCC

felipe.faustino@gmail.com
Luís Antônio dos Santos Silva Universidade Federal de Sergipe (UFS) - PROCC

antonio.silva@dcomp.ufs.br

\author{
Maria Augusta S. N. Nunes \\ Universidade Federal do Estado do \\ Rio de Janeiro (UNIRIO) - PPGI I \\ (UFS) -PROCC \\ gutanunes@uniriotec.br
}

\begin{abstract}
Resumo
Contexto: $O$ desenvolvimento de habilidades socioemocionais pode ser considerado como um elemento bastante relevante a ser incorporado na educação de crianças para o século XXI. A empregabilidade dos mesmos dependerá de como essas habilidades terão sido incorporadas e desenvolvidas durante os processos de ensino-aprendizagem brasileiros atuais, sendo esta uma preocupação recorrente dos gestores a serem, inclusive, apoiadas por meio de políticas públicas. Objetivo: Mapear as iniciativas, buscando evidências científicas, de como o desenvolvimento destas habilidades socioemocionais, tais como Empatia e Mindfulness, tem sido apresentadas, desenvolvidas elou aplicadas às crianças do século XXI integrantes do ensino básico brasileiro com o propósito de auxiliar gestores educacionais. Metodologia: Utilizando o método de Mapeamento Sistemático da Literatura, proposta por Petersen et al., foi realizada uma pesquisa em bases de dados nacionais e internacionais visando responder, dentre outras, à pergunta: "Quais evidências existem que relacionam o uso de tecnologias educacionais digitais/analógicas para o desenvolvimento de habilidades socioemocionais aos estudantes no ensino básico brasileiro?". Resultados: Foram encontrados 96 estudos que apresentam habilidades socioemocionais (Empatia e Mindfulness), que atendem aos critérios de inclusão e exclusão. Estes revelaram que habilidades socioemocionais foram desenvolvidas usando tecnologias educacionais digitais/analógicas e em sua maioria com evidências apoiadas por métodos estatísticos. Conclusão: A análise destas publicações possibilitou obter um panorama sobre as tecnologias educacionais digitais/analógicas que vêm sendo utilizadas no contexto do desenvolvimento de habilidades socioemocionais (Empatia/Mindfulness) no cenário brasileiro e mundial, como e onde têm sido aplicadas, e os resultados obtidos com sua aplicação. Os dados mostram que há, sim, evidências de publicações que desenvolvem habilidades socioemocionais, tais como Empatia e Mindfulness, que são aplicáveis à crianças do século XXI no ensino básico brasileiro, bem como no exterior e, que em sua maioria, são apoiados por métodos/técnicas estatísticas, revelando, assim, rigor na validação de seus resultados.
\end{abstract}

Palavras-Chave: Atenção Plena; Desenvolvimento de Empatia; Educação; Evidências; Empatia; Mindfulness; habilidade socioemocional; Tecnologias Educacionais; Tecnologias Digitais, Crianças, Século XXI.

Cite as: Souza, F. F., Silva, L. A. S., \& Nunes, M. A. S. N. (2020). Evidence on the development of socioemotional skills via digital educational technologies for children of the 21 st century: a systematic mapping of the state of the art as an incentive for managers to support Brazilian public policies (Evidências no desenvolvimento de habilidades socioemocionais via tecnologias educacionais digitais/analógicas para crianças do século XXI: um mapeamento sistemático do estado da arte como fomento a gestores para apoio à políticas públicas brasileiras). Brazilian Journal of Computers in Education (Revista Brasileira de Informática na Educação - RBIE), 28, 1121-1150. DOI: 10.5753/RBIE.2020.28.0.1121 


\begin{abstract}
Context: The development of socioemotional skills can be considered as a very relevant element to be incorporated into the education of children for the 21st century. Their employability will depend on how these skills will have been incorporated and developed during the current Brazilian teaching-learning processes, which is a recurring concern for managers to be even supported through public policies. Objective: To map the initiatives, seeking scientific evidence, of how the development of these socio-emotional skills, such as Empathy and Mindfulness, has been presented, developed and / or applied to children of the 21st century who are part of Brazilian basic education with the purpose of assisting educational managers. Methodology: Using the Systematic Mapping of Literature method, proposed by Petersen et al., A research was carried out in national and international databases aiming to answer, among others, the question: "What evidence exists that relate the use of digital educational technologies? / analogues for the development of socioemotional skills for students in Brazilian basic education? ". Results: 96 studies were found showing socio-emotional skills (Empathy and Mindfulness), which meet the inclusion and exclusion criteria. These revealed that socio-emotional skills were developed using digital / analog educational technologies and mostly with evidence supported by statistical methods. Conclusion: The analysis of these publications made it possible to obtain an overview of the digital / analogue educational technologies that have been used in the context of the development of socioemotional skills (Empathy / Mindfulness) in the Brazilian and worldwide scenario, how and where they have been applied, and the results obtained with your application. The data show that there is evidence of publications that develop socio-emotional skills, such as Empathy and Mindfulness, which are applicable to children of the 21st century in Brazilian basic education, as well as abroad, and which, in their majority, are supported by methods / statistical techniques, thus revealing rigor in the validation of its results.
\end{abstract}

Keywords: Mindfulness; Empathy Development; Education; Evidence; Empathy; Mindfulness; Socio-emotional program; Educational technologies; Digital technologies.

\title{
1 Introdução
}

A Base Nacional Comum Curricular (BNCC) sugere modificações que contemplem as exigências atuais de formação das crianças e jovens brasileiros (MEC, 2019). Sendo que essas diretrizes que guiam os processos de ensino-aprendizagem devem refletir as necessidades mercadológicas e de empregabilidade atuais do século XXI e vindouros. Em seu relatório a Organização das Nações Unidas (ONU), em sintonia com a BNCC, inclui em sua definição de competências, habilidades ligadas a prática cognitiva e socioemocionais para a resolução de "demandas complexas da vida cotidiana, do pleno exercício da cidadania e do mundo do trabalho" no próximo século (KFC, 2016).

Logo, as estratégias e metodologias educacionais atuais devem ser remodeladas para garantir que, além das habilidades básicas (leitura, escrita, cálculo), também, outras, tais como as habilidades transferíveis, bem como, as competências técnico-profissionais, classificadas como habilidades para o século XXI (Resolução de Problemas, Comunicação de Idéias, Criatividade, Liderança e Habilidades Empresariais) sugeridas pela UNESCO (UNESCO, 2016) sejam desenvolvidas nos alunos. Criando assim cidadãos capazes de lidar com as demandas de empregabilidade futuras, tendo em vista o cenário onde o surgimento de novas profissões é certo, onde a maioria das profissões atuais não existirá num intervalo de 10 anos, requerendo assim uma preparação para profissões que ainda surgirão, segundo a Organização de Cooperação e de Desenvolvimento Econômico (OCDE) (Idoeta, 2020).

A OCDE (Idoeta, 2020) corroborando com a UNESCO (UNESCO, 2016), considera que as habilidades do século XXI incluem, além das citadas anteriormente, também, habilidades de Aprendizagem e Inovação (Pensamento Crítico e Resolução de Problemas; Criatividade e Inovação; Comunicação e Colaboração); habilidades de Informação, Mídia e Tecnologia (Alfabetização da Informação, Alfabetização Midiática, Alfabetização das TIC (Informações, Comunicações e Tecnologia)); habilidades de Vida e Carreira (Flexibilidade e Adaptabilidade, Iniciativa e Autodireção, habilidades Sociais e Transculturais, Produtividade e Responsabilidade, Liderança e Responsabilidade) (Fadel,2008). 
Essa visão transformadora da educação e seu papel fundamental para alcançar o desenvolvimento sustentável está contida na Agenda 2030 (ONU, 2020) para o Desenvolvimento Sustentável. A preocupação em promover capacidades que favoreçam o desenvolvimento sustentável é complementada pelo interesse em construir um sentimento de pertencimento que ultrapassa os limites do conceito de nação. Nesse contexto, o desenvolvimento de uma consciência dos vínculos e da interdependência com toda a humanidade é considerado um desafio central, do qual surge a noção de cidadania global, que "se refere a um sentimento de pertencer a uma comunidade mais ampla e a uma humanidade comum. Ele enfatiza a interdependência política, econômica, social e cultural e a interconexão entre o local, o nacional e o global" (UNESCO, 2016).

Dito isso, a Global Citizenship Education (GCE) reconhece três áreas principais de aprendizado no século XXI: cognitiva, socioemocional e comportamental (UNESCO, 2016). Jones \& Doolittle (2017) afirmam o efeito benéfico da aprendizagem das habilidades socioemocionais para crianças e jovens, não só no ambiente escolar, mas também no ambiente de trabalho e em suas relações pessoais e cidadania. Assim, "habilidades socioemocionais são entendidas como a capacidade do indivíduo de refletir sobre suas ações e como elas afetam a si mesmo e aos outros, conhecendo melhor a si mesmo, o indivíduo pode perceber suas melhores habilidades e potencializá-las para obter êxito nas atividades" (Paranhos, 2016, p.30-31).

As principais habilidades socioemocionais, também conhecidas como habilidades do século XXI, por Colagrossi \& Vassimon (2017), são: autoconhecimento, auto regulação, relacionamento pessoal/ habilidades de relacionamento, consciência social e tomada de decisões responsáveis. O desenvolvimento das competências ligadas a essas habilidades, tal como a Empatia, propicia às crianças o entendimento de suas emoções, o foco a atenção e a melhora o relacionamento com os outros. Além disso, técnicas/métodos que apoiem o desenvolvimento das competências ligadas a essas habilidades, tal como o Mindfulness, contribuindo também no desenvolvimento da Empatia (Davis \& Hayes, 2011) impacta positivamente na atenção e relacionamento consigo, reduzindo a depressão, estresse e aumentando o bem-estar (Erbe \& Lohrmann, 2015), e concomitantemente com os outros.

Empatia é a capacidade de poder experimentar a vida psicológica de outra pessoa, projetando-se na outra, para entender o que ela está pensando ou sentindo (Swan \& Riley, 2012). Logo, ela é um alicerce indispensável para relacionamentos saudáveis, pois fornece regulação a interação social (Batson, Lishner, \& Stocks, 2015), previne o bullying (Şahin, 2012), aumenta a habilidade social em pessoas no espectro autista (Cheng et al., 2010), desenvolve cooperação em relação a objetivos compartilhados (De Waal, 2008), regula o estado emocional (Madaliyeva et al., 2015), aumenta a satisfação nos relacionamentos (Long, Angera \& Mikiyasu, 2008), melhora a qualidade da interação professor-aluno (Warren \& Lessner, 2014), aumenta os resultados acadêmicos (Cornelius-White, 2007), e quanto mais presente, menor o nível de agressividade (Garaigordobil, 2012).

Como já dito, a Empatia é utilizada para diferentes fins. O mesmo vale para as técnicas/métodos usados para desenvolvê-la em diferentes contextos e entre essas técnicas está o Mindfulness. Assim como a própria Empatia, os benefícios da prática de Mindfulness englobam também questões como assertividade, regulação emocional, menor reatividade, maior flexibilidade de resposta, habilidades de aconselhamento e inteligência emocional (Davis \& Hayes, 2011). Kaplan (2001) aponta como características desenvolvidas na aplicação de Mindfulness além da Empatia, a compaixão e o altruísmo. Ainda sobre os seus efeitos benéficos, sua prática é responsável pela diminuição do estresse (Ponsoda, 2017) e ajuda ao praticante enxergar a realidade de forma mais clara apoiando a resolução de problemas de forma mais efetiva (Bernárdez, Duran \& Parejo, 2014). 
De forma resumida, a prática de Mindfulness formal pode ser caracterizada por atividades individuais ou em grupo de meditação ou respiração, com o intuito de desenvolver um estado de atenção plena. No entanto, a prática informal utiliza de tarefas do dia-dia e do estado de consciência em sua execução (Bernárdez, Duran \& Parejo, 2014).

Dessa forma, apresenta-se como objetivo deste artigo mapear as iniciativas, buscando-se evidências científicas, de como o desenvolvimento das habilidades socioemocionais, tais como Empatia e Mindfulness, tem sido apresentadas, desenvolvidas e/ou aplicadas às crianças do século XXI integrantes do ensino básico brasileiro. Dentre as iniciativas e evidências mapeadas pesquisam-se àquelas que de alguma forma permeiam as tecnologias educacionais (digitais ou analógicas) e envolvam as habilidades socioemocionais Empatia, Mindfulness, como sugeridas, também pela ONU. Os resultados e as análises conclusivas do artigo servirão como subsídio aos gestores educacionais para a criação de políticas públicas a serem aplicadas ao ensino básico brasileiro.

O artigo está organizado como segue: na Seção 2 apresenta-se a metodologia utilizada; na Seção 3 apresenta-se os resultados obtidos; na Seção 4 os resultados são discutidos; na Seção 5 mostra-se as ameaças à validade; e por fim, as conclusões são apresentadas na Seção 6.

\section{Metodologia}

Este artigo é caracterizado pelo uso do método de Mapeamento Sistemático da Literatura (MSL) proposto por Petersen et al. (2008). Este método é caracterizado por etapas pré-definidas, tais como: (1) definição das questões de pesquisa; (2) identificação dos estudos por meio da escolha das palavras-chave e montagem da string de busca; (3) seleção dos critérios de inclusão e exclusão dos estudos primários, selecionando os estudos relevantes; (4) seguido pela avaliação da qualidade desses estudos; (5) finalmente sintetizando os resultados e analisando-os por meio da discussão.

Para a realização desse MSL foi utilizada a Plataforma Evidências - Módulo SUMARIZE ${ }^{1}$ criado pelo CIEB, facilitando assim o trabalho realizado por três pesquisadores geograficamente distribuídos, onde dois pesquisadores foram responsáveis por selecionar os artigos e enquanto um pesquisador revisava a escolha dos artigos referentes aos dois Subtemas. Foi utilizado, também, materiais lúdicos para o aprendizado e construção do método MSL (Silva et al., 2018) (Série 6Volumes 7, 8, 9 e 10).

\subsection{Questões de Pesquisa}

Este mapeamento buscou responder a seguinte Questão de Pesquisa Primária (QPP): “Quais evidências existem que relacionam o uso de tecnologias educacionais para o desenvolvimento de habilidades socioemocionais no ensino básico brasileiro?". Com objetivo de buscar dados mais aprofundados, bem como a obtenção de dados mais precisos para uso dos gestores, foram

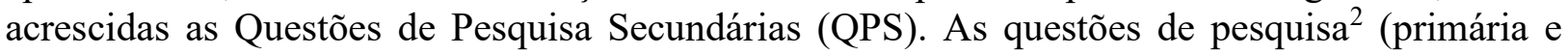

\footnotetext{
${ }^{1}$ https://qeed.nees.com.br/

2 As questões de pesquisa (tanto a primária como as secundárias) têm como objetivo registrar os fatos apresentados no estudo que respondam de forma mais direta possível aos objetivos do estudo. A primária é uma questão mais abrangentes cujos dados extraídos não conseguem ser apresentados/mapeados de forma direta nos estudos, em contrapartidas as questões secundárias por serem mais simples e diretas, conseguem que seus dados extraídos sejam facilmente encontrados.
} 
secundárias) bem como os dados a serem extraídos ${ }^{3}$ propostos para esse mapeamento estão dispostos e selecionados como apresentados na Tabela 1.

Tabela 1: Questões de pesquisa e dados a serem extraídos.

\begin{tabular}{|c|c|}
\hline Questões de Pesquisa & Dados a serem extraídos \\
\hline \multicolumn{2}{|c|}{ Primária } \\
\hline $\begin{array}{c}\text { QPP: Quais evidências existem que relacionam o } \\
\text { uso de tecnologias educacionais para o } \\
\text { desenvolvimento de habilidades socioemocionais } \\
\text { no ensino básico brasileiro? }\end{array}$ & $\begin{array}{c}\text { Evidências da aplicação de tecnologias educacionais } \\
\text { para o desenvolvimento de habilidades } \\
\text { socioemocionais, ferramentas, metodologias, jogos } \\
\text { digitais ou qualquer aplicação de tecnologias } \\
\text { educacionais para o desenvolvimento de habilidades } \\
\text { socioemocionais. }\end{array}$ \\
\hline \multicolumn{2}{|r|}{ Secundárias } \\
\hline $\begin{array}{c}\text { QPS1: Qual o panorama atual das pesquisas sobre } \\
\text { o uso de tecnologias educacionais para o } \\
\text { desenvolvimento de habilidades socioemocionais } \\
\text { no ensino? }\end{array}$ & $\begin{array}{l}\text { Data, tipo e local de publicação (Para os casos } \\
\text { brasileiros a classificação será feita pelo nome do } \\
\text { estado onde ocorreu a coleta da amostra, para o } \\
\text { estrangeiro, será considerado o país) }\end{array}$ \\
\hline $\begin{array}{l}\text { QPS2: A pesquisa informa o cronograma de } \\
\text { execução, bem como, tempo e/ou período } \\
\text { utilizado para realizar a coleta dos dados? }\end{array}$ & Duração, cronograma do estudo. \\
\hline $\begin{array}{l}\text { QPS3: São mencionadas as ameaças à validade e } \\
\text { também como essas ameaças afetam os resultados } \\
\text { e conclusões? }\end{array}$ & Críticas dos próprios autores a seu estudo \\
\hline QPS4: Há algum custo para implementação? & Custo monetário para o estudo \\
\hline QPS5: Qual tipo de escola o estudo é realizado? & Pública ou Particular \\
\hline QPS6: Qual o tipo de intervenção foi realizada? & $\begin{array}{c}\text { Estudo caso-controle (experimental), Estudo } \\
\text { descritivo, Estudo longitudinal, etc. }\end{array}$ \\
\hline QPS7: Qual o tipo de público do estudo? & Alunos, Funcionários, Professores e Pais \\
\hline $\begin{array}{l}\text { QPS8: Qual a técnica/método estatístico usado } \\
\text { para avaliar a evidência? }\end{array}$ & Técnicas/métodos estatísticos utilizados \\
\hline $\begin{array}{l}\text { QPS9: Sobre qual área/subárea a intervenção é } \\
\text { realizada? }\end{array}$ & $\begin{array}{l}\text { Hist. - Empatia Histórica, Socioemocional - } \\
\text { Intervenções na Educação Básica, Socioemocional - } \\
\text { Formação de Professores, etc. }\end{array}$ \\
\hline QPS10: Qual a escolaridade dos participantes? & $\begin{array}{l}\text { Pré-Escola, Creche, Fund. } 1^{\circ} \text { ano, Fund. } 2^{\circ} \text { ano, } \\
\text { Ensino Superior, etc. }\end{array}$ \\
\hline $\begin{array}{l}\text { QPS11: Há o uso de tecnologias educacionais ou } \\
\text { digitais? }\end{array}$ & Tecnologia educacional utilizada no estudo \\
\hline
\end{tabular}

\subsection{Identificação dos estudos primários}

O mapeamento foi conduzido primeiramente nas bases de dados nacionais como SciELO ${ }^{4}$, Educapes $^{5}$, BDComp $^{6}$, LACLO $^{7}$, Plataforma Inovação e Criatividade na Educação Básica ${ }^{8}$, Revista Curriculum Sem Fronteiras ${ }^{9}$, Revista e-curriculum ${ }^{10}$ e Educ@ ${ }^{11}$, além da base de publicações indexadas pela Comissão Especial de Informática na Educação (CEIE) ${ }^{12}$, a saber:

\footnotetext{
${ }^{3}$ Os dados a serem extraídos são os dados apresentados e mapeados dos estudos, de forma mais direta possível que respondam as questões, para que os mesmos, após o preenchimento do protocolo possam ser usados para análise do estudo.

${ }^{4}$ http://scielo.br

${ }^{5}$ https://educapes.capes.gov.br/

6 http://www.lbd.dcc.ufmg.br/bdbcomp/

${ }^{7} \mathrm{http}: / /$ cleilaclo2018.mackenzie.br/pt/index.html

8 http://simec.mec.gov.br/educriativa/mapa_questionario.php

9 http://www.curriculosemfronteiras.org/

$10 \mathrm{https} / / /$ revistas.pucsp.br/curriculum

$11 \mathrm{https}: / / \mathrm{www}$. fcc.org.br/fcc/educ

$12 \mathrm{http}: / /$ www.br-ie.org
} 
Revista Brasileira de Informática na Educação (RBIE), Simpósio Brasileiro de Informática na Educação (SBIE), Anais do Workshop de Informática na Escola (WIE), Anais dos Workshops do Congresso Brasileiro de Informática na Educação (WCBIE), Jornada de Atualização em Informática na Educação e Anais do Workshop de Desafios da Computação Aplicada à Educação. Além dessas bases, também incluímos a base de estudos da revista Renote ${ }^{13}$, importante fonte de estudos na área.

Foram encontrados poucos estudos que abordassem a temática no Brasil, por isso a pesquisa se estendeu também a bases de dados internacionais como ACM Digital Library ${ }^{14}$ e IEEE Xplore Digital Library ${ }^{15}$, além das bases El Compendex ${ }^{16}$, Google Acadêmico ${ }^{17}$, ISI Web of Science $^{18}$, Science@Direct ${ }^{19}$, Scopus ${ }^{20}$ e Springer Link $^{21}$ aplicando ainda como critério que fossem publicados por autores brasileiros, mas devido à pequena quantidade de estudos encontrados, foi aberto o escopo do estudo para estudos publicados também por estrangeiros. Considerando-se o objetivo do estudo bem como a sua complexidade de temas, o mapeamento foi composto por 2 Subtemas distintos dentro do contexto socioemocional: Subtema 1 (Empatia) e Subtema 2 (Mindfulness).

\subsubsection{Termos utilizados na busca}

Presente na ferramenta utilizada (SUMARIZE- CIEB), a definição dos termos de pesquisa seguiram a estratégia PICOC (População, Intervenção, Comparação, Resultado/Outcome e Contexto)(Kitchenham \& Charters, 2007), dados estes apresentados para cada Subtema na Tabela 2.

Tabela 2: População, Intervenção, Comparação, Resultado e Contexto.

\begin{tabular}{|c|c|c|}
\hline PICOC & Subtema 1 (Empatia) & Subtema 2 (Mindfulness) \\
\hline População & \multicolumn{2}{|c|}{ crianças, adolescentes, jovem } \\
\hline Intervenção & $\begin{array}{c}\text { treinamento de empatia,estimular } \\
\text { empatia, treinamento empático, } \\
\text { entendimento empático, empatia, } \\
\text { desenvolvimento de empatia, } \\
\text { desenvolvimento empático, } \\
\text { crescimento empático; }\end{array}$ & Mindfulness, meditação, atenção plena \\
\hline Comparaçåo & \multicolumn{2}{|c|}{ antes e após utilização das tecnologias educacionais digitais } \\
\hline Resultado & $\begin{array}{c}\text { soft skills, aprendizagem } \\
\text { socioemocional, melhoria de } \\
\text { empatia }\end{array}$ & atenção,soft skills, aprendizagem socioemocional \\
\hline Contexto & \multicolumn{2}{|c|}{ escola, educação básica, aprendizagem, ensino, aula } \\
\hline
\end{tabular}

Para a busca, foram considerados os estudos e soluções que possuíssem os termos listados na Tabela 3 para cada MSL e seus respectivos sinônimos em seus títulos, resumos ou palavras-

\footnotetext{
$13 \mathrm{http}: / /$ seer.ufrgs.br/renote/

14 http://portal.acm.org

$15 \mathrm{http}$ ://ieeexplore.ieee.org

$16 \mathrm{https} / / / \mathrm{www}$. elsevier.com/solutions/engineering-village/content/compendex

${ }^{17} \mathrm{http}: / /$ scholar.google.com

18 http://www.isiknowledge.com

$19 \mathrm{http}: / / \mathrm{www} \cdot$.sciencedirect.com

$20 \mathrm{http}: / / \mathrm{www}$. scopus.com

$21 \mathrm{http}: / /$ link.springer.com
} 
chaves. Para as bases internacionais foram utilizadas as respectivas traduções inicialmente limitando a busca por trabalhos publicados por autores brasileiros e/ou desenvolvidos no Brasil. Limitação desconsiderada tendo em vista o pouco número de ocorrências, assim também abrangendo os estudos estrangeiros ${ }^{22}$ com aplicação em países diferentes do Brasil.

Tabela 3: Palavras-chave, sinônimos e termos em inglês utilizados nas buscas.

\begin{tabular}{|c|c|c|c|}
\hline Subtemas & Palavras-chave & Sinônimos & $\begin{array}{c}\text { Termos correspondente em } \\
\text { Inglês }\end{array}$ \\
\hline \multirow{4}{*}{ Subtema 1 (Empatia) } & Empatia & $\begin{array}{c}\text { empatia afetiva, } \\
\text { empatia cognitiva, } \\
\text { empático, entendimento } \\
\text { empático, habilidade } \\
\text { empática, regulação } \\
\text { emocional, treinamento } \\
\text { de empatia, treinamento } \\
\text { empático }\end{array}$ & $\begin{array}{c}\text { affective empathy, cognitive } \\
\text { empathy, emotional } \\
\text { regulation, empathic, } \\
\text { empathic skill, empathic } \\
\text { training, empathic } \\
\text { understanding, empathy, } \\
\text { empathy training }\end{array}$ \\
\cline { 2 - 4 } & Estimular empatia & crescimento da \\
empatia, crescimento \\
empático, \\
desenvolvimento de \\
empatia, \\
\end{tabular}

Na Tabela 4, estão os termos que são compartilhados por cada MSL e seus respectivos sinônimos em seus títulos, resumos ou palavras-chaves.

Tabela 4: Palavras-chave, sinônimos e termos em inglês utilizados nas buscas por todos os MSL.

\begin{tabular}{|c|c|c|}
\hline Palavras-chave & Sinônimos & Termos correspondente em Inglês \\
\hline Educação & $\begin{array}{c}\text { Ensino, Escola, aprendizagem, } \\
\text { sala de aula }\end{array}$ & $\begin{array}{c}\text { School, Education, classroom, } \\
\text { learning, teaching }\end{array}$ \\
\hline
\end{tabular}

Assim, foram geradas strings de busca (Tabela 5) correspondentes a cada Subtema deste mapeamento:

Tabela 5: Strings de busca de cada Subtema.

\begin{tabular}{|c|c|c|}
\hline Temas & Português & Inglês \\
\hline Subtema 1 & ("Educação" OR "Ensino" OR "Escola" & ( "Education" OR "classroom" OR "learning" \\
(Empatia) & OR "aprendizagem" OR "sala de aula") & OR "school" OR "teaching") AND ( "affective \\
& AND ("empatia" OR "empatia afetiva" & empathy" OR "cognitive empathy" OR \\
& OR "empatia cognitiva" OR "empático" & "emotional regulation" OR "empathic" OR \\
& OR "entendimento empático" OR & "empathic skill" OR "empathic training" OR \\
& "habilidade empática" OR "regulação & "empathic understanding" OR "empathy" OR \\
& $\begin{array}{c}\text { emocional" OR "treinamento de empatia" } \\
\text { OR "treinamento empático") AND } \\
\end{array}$ & $\begin{array}{c}\text { "empathy training") AND ( "empathic } \\
\text { development" OR "empathic growth" OR } \\
\end{array}$ \\
& $\begin{array}{c}\text { estimular empatia" OR "crescimento da } \\
\text { empatia" OR "crescimento empático" OR } \\
\text { "desenvolvimento de empatia" OR }\end{array}$ & $\begin{array}{c}\text { "empathic improvement" OR "empathy } \\
\text { development" OR "empathy growth" OR }\end{array}$ \\
\hline
\end{tabular}

22 Estudos estrangeiros são aqueles em que o primeiro autor não é brasileiro 


\begin{tabular}{|c|c|c|}
\hline & $\begin{array}{c}\text { "desenvolvimento empático" OR } \\
\text { "melhoramento empático" OR "melhoria } \\
\text { de empatia") }\end{array}$ & $\begin{array}{c}\text { "empathy improvement" OR "stimulate } \\
\text { empathy") }\end{array}$ \\
\hline $\begin{array}{c}\text { Subtema } 2 \\
\text { (Mindfulness) }\end{array}$ & $\begin{array}{l}\text { ("Mindfulness" OR "atenção plena" OR } \\
\text { "meditação" OR "mindful") AND } \\
\text { ("habilidade socioemocional" OR } \\
\text { "habilidades socioemocionais" OR } \\
\text { "competência socioemocional" OR } \\
\text { "competências socioemocionais" OR } \\
\text { "aprendizagem socioemocional") AND } \\
\text { ("Educação" OR "Educação Básica" OR } \\
\text { "Ensino" OR "Escola" OR } \\
\text { "aprendizagem" OR "sala de aula") }\end{array}$ & $\begin{array}{l}\text { ("Mindfulness" OR "meditation" OR "mindful") } \\
\text { AND ("Socio-emotional skill” OR “socio- } \\
\text { emotional characteristic" OR “non-cognitive } \\
\text { skill OR "soft skill”) AND ("school" OR } \\
\text { "Education" OR "classroom" OR "learning" OR } \\
\text { "teaching") }\end{array}$ \\
\hline
\end{tabular}

As buscas foram construídas com operadores booleanos OR e AND entre os termos buscado e seus sinônimos. Para ampliar a busca se fez necessária a utilização do operador coringa (*) nas palavras com variações nas bases que davam suporte a esse operador. As buscas foram conduzidas em 31 de outubro de 2019.

\subsection{Critérios de Seleção}

O processo de seleção de publicações foi composto por duas etapas, chamadas filtros. No primeiro filtro, os pesquisadores realizaram a leitura somente do título e do abstract relacionadas à intervenções com o objetivo de desenvolver habilidades socioemocionais, aplicando os critérios de inclusão (CI) e exclusão (CE) listados na Tabela 6 aplicadas a cada um dos Subtemas. No segundo filtro, os pesquisadores realizaram a leitura completa das publicações selecionadas no primeiro filtro, sendo submetidos aos mesmos critérios de inclusão e exclusão.

Tabela 6: Critérios de inclusão (CI) e Critérios de exclusão (CE).

\begin{tabular}{|c|c|}
\hline ID & Critério de inclusão \\
\hline CI1 & Esteja acessível via web de maneira gratuita \\
\hline CI2 & Estudos publicados no idioma Português e Inglês \\
\hline CI3 & Estudos Primários e Secundários \\
\hline CI4 & Critério de exclusão \\
\hline \# & estudos duplicados \\
\hline CE1 & Trabalhos publicados com data inferior a 2009 \\
\hline CE2 & Trabalhos que não contemplam a temática \\
\hline CE3 & Trabalhos que não estavam disponíveis para visualização do texto \\
\hline CE4 &
\end{tabular}

Para cada etapa a ferramenta SUMARIZE permitiu a avaliação desse processo por pares de pesquisadores, assim minimizando as chances da eliminação ou seleção de estudos que não contemplem os CI e CE. Nos casos em conflito, o SUMARIZE só concluía a avaliação com a análise de um terceiro pesquisador.

No terceiro filtro, as publicações passaram por um conjunto de questões que foram criadas para uma avaliação de qualidade dos estudos. Para que fossem respondidas foi necessária a leitura completa da publicação/artigo. As Questões de Qualidade (QQ) são apresentadas na Tabela 7 com as opções de resposta e sua pontuação para cada pergunta. A Pontuação de Avaliação de Qualidade é resultado da soma da pontuação de cada pergunta, sendo a Pontuação Máxima $(10,0)$ que o estudo poderia alcançar após essa soma e uma Pontuação de Corte $(7,0)$ na qual a publicação que ficou com pontuação menor ou igual a ela, foi rejeitado (etapa gerenciada pelo SUMARIZE). 
Tabela 7: Questões de Qualidade, Opções de Resposta e a Pontuação.

\begin{tabular}{|c|c|}
\hline Questões de Qualidade & Opções de Resposta \\
\hline $\begin{array}{l}\text { QQ1:São mencionadas as ameaças à validade e } \\
\text { também como essas ameaças afetam os resultados } \\
\text { e conclusões? }\end{array}$ & $\begin{array}{ll}\text { - } & \text { Sim }(1,0) \\
\text { - } & \text { Parcialmente }(0,5) \\
\text { - } & \text { Não }(0,0) \\
\end{array}$ \\
\hline $\begin{array}{l}\text { QQ2: O estudo apresenta técnica/método } \\
\text { estatístico para avaliar as evidências? }\end{array}$ & $\begin{array}{ll}- & \text { Sim }(1,0) \\
- & \text { Parcialmente }(0,5) \\
- & \text { Não }(0,0) \\
\end{array}$ \\
\hline $\begin{array}{l}\text { QQ3: O estudo descreve o custo para } \\
\text { implementação? }\end{array}$ & $\begin{array}{ll}\text { - } & \text { Sim }(1,0) \\
\text { - } & \text { Não }(0,0) \\
\end{array}$ \\
\hline $\begin{array}{l}\text { QQ4: Houve a descrição ou referência a estudos } \\
\text { similares pelos autores? }\end{array}$ & $\begin{array}{ll}\text { - } & \text { Sim }(1,0) \\
\text { - } & \text { Não }(0,0) \\
\end{array}$ \\
\hline $\begin{array}{l}\text { QQ5: O estudo descreve de forma clara o método } \\
\text { aplicado? }\end{array}$ & $\begin{array}{ll}- & \text { Sim }(1,0) \\
- & \text { Parcialmente }(0,5) \\
\text { - } & \text { Não }(0,0) \\
\end{array}$ \\
\hline $\begin{array}{l}\text { QQ6: Os resultados foram capazes de atender o } \\
\text { objetivo do estudo? }\end{array}$ & $\begin{array}{ll} & \text { Sim }(1,0) \\
\text { - } & \text { Parcialmente }(0,5) \\
\text { - } & \text { Não }(0,0) \\
\end{array}$ \\
\hline $\begin{array}{c}\text { QQ7: O trabalho informa o cronograma de } \\
\text { execução? }\end{array}$ & $\begin{array}{ll}- & \text { Sim }(1,0) \\
- & \text { Parcialmente }(0,5) \\
-\quad & \text { Não }(0,0) \\
\end{array}$ \\
\hline $\begin{array}{l}\text { QQ8: Há o uso de tecnologias educacionais ou } \\
\text { digitais de forma clara? }\end{array}$ & $\begin{array}{ll} & \text { Sim }(1,0) \\
- & \text { Parcialmente }(0,5) \\
- & \text { Não }(0,0) \\
\end{array}$ \\
\hline $\begin{array}{l}\text { QQ9: O estudo atribui o desenvolvimento de } \\
\text { Habilidades Socioemocionais como resultado do } \\
\text { experimento? }\end{array}$ & $\begin{array}{ll}\text { - } & \text { Sim }(2,0) \\
\text { - Não }(0,0)\end{array}$ \\
\hline
\end{tabular}

Assim reduzindo para um total de 96 estudos conforme apresentados na Tabela 8 .

Tabela 8: Detalhamento do Processo de Seleção dos estudos.

\begin{tabular}{|c|c|c|c|c|c|c|}
\hline \multirow[t]{2}{*}{ Bases } & \multicolumn{2}{|c|}{ Estudos Primários } & \multicolumn{2}{|c|}{$\begin{array}{c}\text { Estudos Relevantes } \\
\text { Selecionados após CI e CE }\end{array}$} & \multirow{2}{*}{$\begin{array}{c}\text { Total de } \\
\text { Estudos } \\
\text { Relevantes } \\
\text { após CI e CE }\end{array}$} & \multirow{2}{*}{$\begin{array}{c}\text { Estudos } \\
\text { Relevantes } \\
\text { após Questões } \\
\text { de Qualidade }\end{array}$} \\
\hline & Empatia & Mindfulness & Empatia & Mindfulness & & \\
\hline Scielo & 268 & 4 & 6 & 2 & 8 & 5 \\
\hline $\begin{array}{c}\text { Google } \\
\text { Acadêmico }\end{array}$ & 1680 & 92 & 63 & 21 & 84 & 14 \\
\hline $\begin{array}{l}\text { Revista e- } \\
\text { curriculum }\end{array}$ & 0 & 0 & 0 & 0 & 0 & 0 \\
\hline $\begin{array}{l}\text { Plataforma } \\
\text { Inovação e } \\
\text { Criatividade } \\
\text { na Educação } \\
\text { Básica }\end{array}$ & 0 & 0 & 0 & 0 & 0 & 0 \\
\hline $\begin{array}{c}\text { Revista } \\
\text { Currículo Sem } \\
\text { Fronteiras }\end{array}$ & 0 & 0 & 0 & 0 & 0 & 0 \\
\hline CEIE & 3 & 2 & 1 & 2 & 3 & 2 \\
\hline Renote & 3 & 0 & 0 & 0 & 0 & 0 \\
\hline EDUC@ & 43 & 0 & 0 & 0 & 0 & 0 \\
\hline LACLO & 0 & 0 & 0 & 0 & 0 & 0 \\
\hline BDBComp & 1 & 0 & 0 & 0 & 0 & 0 \\
\hline Educapes & 162 & 0 & 9 & 0 & 9 & 3 \\
\hline Scopus & 76 & 3 & 0 & 2 & 2 & 1 \\
\hline $\mathrm{ACM}$ & 229 & 46 & 15 & 18 & 33 & 8 \\
\hline IEEE & 243 & 278 & 23 & 16 & 39 & 10 \\
\hline Science Direct & 2249 & 31 & 80 & 3 & 83 & 44 \\
\hline
\end{tabular}




\begin{tabular}{|c|c|c|c|c|c|c|}
\hline Springer Link & 374 & 0 & 28 & 0 & 28 & 3 \\
\hline PEPSIC & 118 & 0 & 5 & 0 & 5 & 5 \\
\hline $\begin{array}{c}\text { Web of } \\
\text { Science }\end{array}$ & 56 & 4 & 5 & 2 & 7 & 1 \\
\hline $\begin{array}{c}\text { El } \\
\text { Compendex }\end{array}$ & 96 & 0 & 0 & 0 & 0 & 0 \\
\hline Subtotal & $\mathbf{4 1 0 4}$ & $\mathbf{4 6 0}$ & $\mathbf{2 3 5}$ & $\mathbf{6 6}$ & $\mathbf{3 0 1}$ & $\mathbf{9 6}$ \\
\hline
\end{tabular}

Após essas etapas, decidiu-se incluir estudos que focam no Ensino Superior, já que o Subtema 2 (Mindfulness) possui poucos estudos focados no ensino básico, por essa ser uma área recente. Logo estudos que tem como foco o Ensino Superior, estão incluídos neste total de 96 estudos selecionados e foram incluídos para mostrar estudos que poderiam ser aplicados no ensino básico, sendo adaptados conforme a realidade da escola e do ensino básico. Tentou-se fazer o mesmo procedimento que foi feito no Subtema 1 (Empatia) para o Subtema 2 (Mindfulness), mas devido a alta quantidade de estudos foi necessária a exclusão de estudos secundários estrangeiros e de estudos com participantes de nível superior que não tinham como foco a área/subárea "Socioemocional - Formação de Professores" listada na QPS 10.

\section{Resultados}

Os 96 estudos selecionados estão disponíveis em uma planilha com acesso pelo link seguinte https://docs.google.com/spreadsheets/d/1pFt5-

1IkHU_XoXGQg4xflPYZXmgSGmpYqqaEUMzfAAw/edit?usp=sharing, onde é possível visualizar a referência completa do estudo e um código de identificação, (EE) para os Estudos de Empatia e (EM) para os Estudos de Mindfulness, acrescido de um valor numérico, que será utilizado como referência, assim facilitando o entendimento dos resultados obtidos.

A seguir, as análises realizadas para responder às questões de pesquisa secundárias apresentadas anteriormente, já que a questão primária de pesquisa será respondida no tópico de Discussão. Cada QP traz as respostas referentes a ambos os Subtemas, como identificado, com exceção da QPS1, que aborda o panorama geral. Nas tabelas os estudos estão divididos entre estudos brasileiros $^{23}$ e estudos estrangeiros ${ }^{24}$.

\section{QPS1: Qual o panorama atual das pesquisas sobre o uso de tecnologias educacionais para o desenvolvimento de habilidades socioemocionais no ensino?}

Foram encontradas pesquisas em torno de ambos os Subtemas no Brasil conforme Tabela 9. Em relação a Empatia os 12 estudos foram distribuídos em diferentes estados, sendo São Paulo (4) e Minas Gerais (3) com a maior quantidade desses estudos. Sobre o Subtema Mindfulness foram encontrados 3 estudos brasileiros, todos aplicados no Rio Grande do Sul.

Tabela 9: Estudos Brasileiros Selecionados por Estado referentes ao Subtema 1 (Empatia) e Subtema 2 (Mindfulness).

\begin{tabular}{|c|c|c|c|}
\hline Estado & Estudos Brasileiros (Empatia) & $\begin{array}{c}\text { Estudos Brasileiros } \\
\text { (Mindfulness) }\end{array}$ & Quantitativo de Estudos \\
\hline São Paulo & EE64, EE73, EE76, EE78 & - & 04 \\
\hline Minas Gerais & EE10, EE69, EE71 & - & 03 \\
\hline Paraná & EE63, EE75 & - & 02 \\
\hline Rio de Janeiro & EE74 & - & 01 \\
\hline Distrito Federal & EE77 & - & 01 \\
\hline Santa Catarina & EE65 & EM09, EM12, EM16 & 01 \\
\hline Rio Grande do Sul & - & 03 \\
\hline
\end{tabular}

${ }^{23}$ Estudos em que o primeiro autor é brasileiro e/ou seu local coleta foi o Brasil

24 Estudos em que o primeiro autor não é brasileiro e/ou seu local coleta não foi o Brasil 
Em relação aos dados coletados envolvendo estudos estrangeiros para o Subtema 1 (Empatia) destacam-se os Estados Unidos (14), Turquia (9) e a Espanha (8) em quantidade de estudos conforme apresentado na Tabela 10, que classifica os estudos estrangeiros pelo país em que foi realizado. Para o Subtema 2 (Mindfulness), 14 dos 17 estudos selecionados são estrangeiros com destaque para Portugal (5), também foram encontrados trabalhos na Espanha (2) e China (2), além disso países como Austrália, França, Israel, Estados Unidos e Japão apresentaram apenas um estudo cada.

Tabela 10: Estudos Selecionados por País referentes ao Subtema 1 (Empatia) e Subtema 2 (Mindfulness).

\begin{tabular}{|c|c|c|c|}
\hline País & $\begin{array}{l}\text { Estudos Estrangeiros } \\
\text { (Empatia) }\end{array}$ & $\begin{array}{l}\text { Estudos Estrangeiros } \\
\text { (Mindfulness) }\end{array}$ & $\begin{array}{l}\text { Quantitativo de } \\
\text { Estudos }\end{array}$ \\
\hline $\begin{array}{l}\text { Estados } \\
\text { Unidos }\end{array}$ & $\begin{array}{c}\text { EE03, EE07, EE13, EE20, EE23, EE35, } \\
\text { EE38, EE39, EE49, EE56, EE60, EE62, } \\
\text { EE72, EE79 }\end{array}$ & EM07 & 15 \\
\hline Turquia & $\begin{array}{c}\text { EE04, EE09, EE34, EE37, EE40, EE41, } \\
\text { EE61, EE67, EE70 }\end{array}$ & - & 09 \\
\hline Espanha & $\begin{array}{l}\text { EE11, EE19, EE25, EE29, EE30, EE31, } \\
\text { EE50, EE51 }\end{array}$ & EM04, EM06 & 10 \\
\hline Israel & EE27, EE33, EE36, EE57, EE58 & EM14 & 06 \\
\hline Holanda & EE06, EE08, EE18, EE52 & - & 04 \\
\hline $\begin{array}{c}\text { Coréia do } \\
\text { Sul } \\
\end{array}$ & EE24, EE46, EE53 & - & 03 \\
\hline Itália & EE17, EE22, EE47 & - & 03 \\
\hline Alemanha & EE26, EE28 & - & 02 \\
\hline Grécia & EE16, EE42 & - & 02 \\
\hline Austrália & EE01, EE54 & EM11 & 03 \\
\hline Suiça & EE21, EE68 & - & 02 \\
\hline Jordânia & EE66 & - & 01 \\
\hline Canadá & EE02 & - & 01 \\
\hline Romênia & EE59 & - & 01 \\
\hline Malta & EE43 & - & 01 \\
\hline Inglaterra & EE55 & - & 01 \\
\hline Reino Unido & EE26 & - & 01 \\
\hline Portugal & EE14 & $\begin{array}{l}\text { EM01, EM02, EM10, } \\
\text { EM14, EM15 }\end{array}$ & 06 \\
\hline Taiwan & EE15 & - & 01 \\
\hline Lituânia & EE12 & - & 01 \\
\hline Cazaquistão & EE05 & - & 01 \\
\hline Barbados & EE45 & - & 01 \\
\hline $\begin{array}{l}\text { Nova } \\
\text { Zelândia }\end{array}$ & EE32 & - & 01 \\
\hline Rússia & EE48 & - & 01 \\
\hline Irlanda & EE44 & - & 01 \\
\hline China & - & EM08, EM17 & 02 \\
\hline França & - & EM03 & 01 \\
\hline Japão & - & EM05 & 01 \\
\hline
\end{tabular}

O escopo do MSL é formado por estudos de 2009 a 2019, conforme a Figura 1. Em relação a Empatia, a um aumento de estudos comparando o ano de 2009 (4) a 2019 (12). Nota-se então um aumento do interesse no desenvolvimento de Empatia na comunidade científica.

Os estudos encontrados sobre Mindfulness se distribuem entre os anos de 2014 e 2018. Sendo os anos de 2016, 2017 e 2018 com 5 estudos publicados cada como apresentado na Figura 1. Assim demonstrando um interesse crescente pela prática de Mindfulness no contexto escolar. A ausência de publicações relevantes no ano de 2019 pode ser compreendida pelo fato das buscas 
terem sido feitas antes de seu término, logo, não podemos afirmar a inexistência de estudos relevantes em 2019.

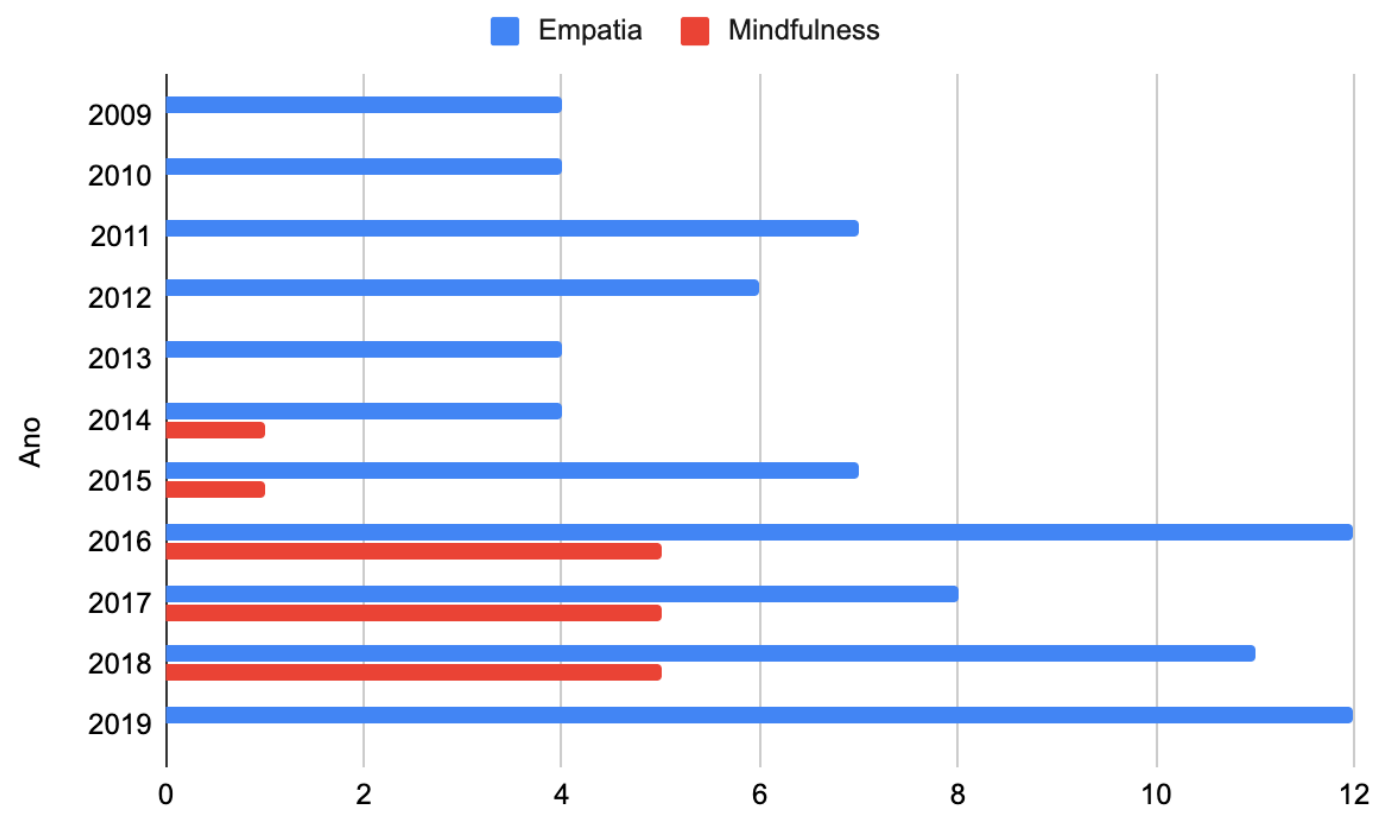

Figura 1: Distribuição dos Estudos Selecionados por Data de Publicação

Abaixo, é apresentado na Tabela 11 informações referentes a quantidade e identificação desses estudos em referência ao ano.

Tabela 11: Distribuição dos Estudos Selecionados por Ano de publicação Subtema 1 (Empatia) e Subtema 2 (Mindfulness).

\begin{tabular}{|c|c|c|c|c|c|}
\hline Ano & $\begin{array}{l}\text { Estudos Estrangeiros } \\
\text { (Empatia) }\end{array}$ & $\begin{array}{c}\text { Estudos } \\
\text { Brasileiros } \\
\text { (Empatia) }\end{array}$ & $\begin{array}{c}\text { Estudos } \\
\text { Estrangeiros } \\
\text { (Mindfulness) }\end{array}$ & $\begin{array}{c}\text { Estudos } \\
\text { Brasileiros } \\
\text { (Mindfulness) }\end{array}$ & $\begin{array}{l}\text { Quantitativo de } \\
\text { Estudos }\end{array}$ \\
\hline 2009 & EE09, EE30, EE37 & EE63 & - & - & 04 \\
\hline 2010 & EE01, EE13, EE15 & EE10 & - & - & 04 \\
\hline 2011 & $\begin{array}{l}\text { EE02, EE17, EE26, } \\
\text { EE35, EE46, EE61 }\end{array}$ & EE69 & - & - & 07 \\
\hline 2012 & $\begin{array}{c}\text { EE31, EE32, EE40, } \\
\text { EE47, EE59 }\end{array}$ & EE71 & - & - & 06 \\
\hline 2013 & $\begin{array}{c}\text { EE20, EE22, EE25, } \\
\text { EE53 }\end{array}$ & - & - & - & 04 \\
\hline 2014 & $\begin{array}{l}\text { EE19, EE24, EE34, } \\
\text { EE41 }\end{array}$ & - & EM04 & - & 05 \\
\hline 2015 & $\begin{array}{l}\text { EE05, EE14, EE16, } \\
\text { EE38, EE52, EE68 }\end{array}$ & EE75 & - & EM16 & 08 \\
\hline 2016 & $\begin{array}{l}\text { EE07, EE08, EE27, } \\
\text { EE28, EE42, EE44, } \\
\text { EE48, EE50, EE70, } \\
\text { EE79 }\end{array}$ & EE77, EE78 & $\begin{array}{c}\text { EM03, EM07, } \\
\text { EM08, EM14, } \\
\text { EM17 }\end{array}$ & - & 17 \\
\hline 2017 & $\begin{array}{c}\text { EE43, EE51, EE55, } \\
\text { EE62, } \\
\text { EE72 }\end{array}$ & $\begin{array}{l}\text { EE65, EE74, } \\
\text { EE76 }\end{array}$ & $\begin{array}{c}\text { EM01, EM05, } \\
\text { EM06, EM10, } \\
\text { EM11 }\end{array}$ & - & 13 \\
\hline 2018 & $\begin{array}{l}\text { EE12, EE18, EE21, } \\
\text { EE29, EE33, EE36, } \\
\text { EE45, EE54, EE56, } \\
\text { EE66 }\end{array}$ & EE64 & $\begin{array}{c}\text { EM02, EM13, } \\
\text { EM15 }\end{array}$ & EM09, EM12 & 16 \\
\hline 2019 & $\begin{array}{l}\text { EE03, EE04, EE06, } \\
\text { EE11, EE23, EE39, }\end{array}$ & EE73 & - & - & 12 \\
\hline
\end{tabular}




\begin{tabular}{|c|c|c|c|c|c|}
\hline & $\begin{array}{c}\text { EE49, EE57, EE58, } \\
\text { EE60, EE67 }\end{array}$ & & & & \\
\hline Total & $\mathbf{6 7}$ & $\mathbf{1 2}$ & $\mathbf{1 4}$ & $\mathbf{3}$ & $\mathbf{9 6}$ \\
\hline
\end{tabular}

Quanto aos dados analisados em relação aos estudos de Empatia e conforme a Tabela 12, houveram estudos publicados em periódico (59), conferência (14), congresso (2) e simpósio (1); assim como dissertações de mestrado (3). Desses estudos 12 foram realizados no Brasil, sendo periódico (9) e dissertações de mestrado (3). Nota-se o bom espaço dado a estudos relacionados ao tema em periódicos e em como o tema é bastante discutido na comunidade científica.

Tabela 12: Distribuição dos Estudos Selecionados por Tipo de Publicação para o Subtema 1 (Empatia) e Subtema 2 (Mindfulness).

\begin{tabular}{|c|c|c|c|c|c|}
\hline $\begin{array}{c}\text { Tipo de } \\
\text { Publicação }\end{array}$ & $\begin{array}{l}\text { Estudos Estrangeiros } \\
\text { (Empatia) }\end{array}$ & $\begin{array}{c}\text { Estudos } \\
\text { Brasileiros } \\
\text { (Empatia) } \\
\end{array}$ & $\begin{array}{c}\text { Estudos } \\
\text { Estrangeiros } \\
\text { (Mindfulness) } \\
\end{array}$ & $\begin{array}{c}\text { Estudos } \\
\text { Brasileiros } \\
\text { (Mindfulness) } \\
\end{array}$ & $\begin{array}{l}\text { Quantitativo } \\
\text { de Estudos }\end{array}$ \\
\hline Conferência & $\begin{array}{c}\text { EE01, EE06, EE13, } \\
\text { EE16, EE17, } \\
\text { EE18, EE19, EE28, } \\
\text { EE37, EE42, } \\
\text { EE48, EE59, EE68, EE79 }\end{array}$ & - & - & - & 14 \\
\hline Congresso & EE21, EE43 & - & $\begin{array}{c}\text { EM04, EM05, } \\
\text { EM07 } \\
\end{array}$ & EM09, EM16 & 07 \\
\hline $\begin{array}{l}\text { Dissertação } \\
\text { de Mestrado }\end{array}$ & - & $\begin{array}{l}\text { EE63, EE64, } \\
\text { EE65 }\end{array}$ & $\begin{array}{c}\text { EM01, EM02, } \\
\text { EM10, EM13, } \\
\text { EM15 }\end{array}$ & EM12 & 09 \\
\hline Periódico & $\begin{array}{l}\text { EE02, EE03, EE04, } \\
\text { EE05, EE07, EE08, } \\
\text { EE09, EE11, EE12, } \\
\text { EE14, EE15, EE20, } \\
\text { EE22, EE23, EE25, } \\
\text { EE26, EE27, EE29, } \\
\text { EE30, EE31, EE32, } \\
\text { EE33, EE34, EE35, } \\
\text { EE36, EE38, EE39, } \\
\text { EE40, EE41, EE44, } \\
\text { EE45, EE46, EE47, } \\
\text { EE49, EE50, EE51, } \\
\text { EE52, EE53, EE54, } \\
\text { EE55, EE56, EE57, } \\
\text { EE58, EE60, EE61, } \\
\text { EE62, EE66, EE67, } \\
\text { EE70, EE72 }\end{array}$ & $\begin{array}{l}\text { EE10, EE69, } \\
\text { EE71, EE73, } \\
\text { EE74, EE75, } \\
\text { EE76, EE77, } \\
\text { EE78 }\end{array}$ & $\begin{array}{l}\text { EM03, EM06, } \\
\text { EM08, EM11, } \\
\text { EM14, EM } 17\end{array}$ & - & 65 \\
\hline Simpósio & EE24 & - & - & - & 01 \\
\hline Total & 67 & 12 & 14 & 3 & 96 \\
\hline
\end{tabular}

No tocante aos estudos referentes a Mindfulness tem-se a concentração em publicações em congressos (5), dissertações de mestrado (6) e em periódicos (6). Os achados nacionais contemplam um de cada tipo previamente mencionado, como disposto na Tabela 12.

QPS2 - A pesquisa informa o cronograma de execução, bem como, tempo e/ou período utilizado para realizar a coleta dos dados?

\section{Empatia}

Em relação a Empatia 12 estudos não apresentaram de forma clara o cronograma de execução e 67 apresentaram cronograma. $\mathrm{O}$ tempo do experimento variou entre alguns encontros semanais 
(EE01, EE06) a meses (EE03, EE20) de estudo. Logo abaixo, a Tabela 13 mostra informações sobre os estudos:

Tabela 13: Estudos Selecionados por Informação do Cronograma de Execução referentes ao Subtema 1 (Empatia).

\begin{tabular}{|c|c|c|c|}
\hline Cronograma & Estudos Estrangeiros & Estudos Brasileiros & $\begin{array}{l}\text { Quantitativo de } \\
\text { Estudos }\end{array}$ \\
\hline $\begin{array}{l}\text { Informaram } \\
\text { Cronograma } \\
\text { de Execução }\end{array}$ & $\begin{array}{l}\text { EE02, EE03, EE04, EE05, EE07, EE09, } \\
\text { EE11, EE12, EE13, EE14, EE15, EE16, } \\
\text { EE17, EE19, EE20, EE22, EE23, EE25, } \\
\text { EE26, EE27, EE28, EE29, EE30, EE31, } \\
\text { EE32, EE33, EE34, EE35, EE36, EE37, } \\
\text { EE38, EE39, EE40, EE41, EE42, EE43, } \\
\text { EE44, EE45, EE46, EE48, EE49, EE50, } \\
\text { EE51, EE52, EE53, EE54, EE55, EE56, } \\
\text { EE57, EE58, EE59, EE60, EE61, EE62, } \\
\text { EE66, EE67, EE68, EE70, EE72, EE79 }\end{array}$ & $\begin{array}{c}\text { EE10, EE64, EE65, } \\
\text { EE71, } \\
\text { EE74, EE76, } \\
\text { EE77 }\end{array}$ & 67 \\
\hline $\begin{array}{c}\text { Não } \\
\text { Informaram } \\
\text { Cronograma } \\
\text { de Execução } \\
\end{array}$ & $\begin{array}{c}\text { EE01, EE06, EE08, EE18, } \\
\text { EE21, EE24, EE47 }\end{array}$ & $\begin{array}{c}\text { EE63, EE69, EE73, } \\
\text { EE75, EE78 }\end{array}$ & 12 \\
\hline Total & 67 & 12 & 79 \\
\hline
\end{tabular}

\section{Mindfulness}

Dos 17 estudos selecionados apenas 5 não apresentaram cronograma de execução como disposto na Tabela 14. Dos que informaram o cronograma, o tempo do experimento variou de 5 dias (EM05) a dois anos (EM14). A grande maioria descreve o cronograma em conjunto com algum protocolo de aplicação Mindfulness (EM1, EM2, EM3, EM6, EM9, EM12). Esses protocolos se estendem geralmente por 8 semanas.

Tabela 14: Estudos Selecionados por Informação do Cronograma de Execução referentes ao Subtema 2 (Mindfulness).

\begin{tabular}{|c|c|c|c|}
\hline Cronograma & Estudos Estrangeiros & Estudos Brasileiros & $\begin{array}{c}\text { Quantitativo de } \\
\text { Estudos }\end{array}$ \\
\hline $\begin{array}{l}\text { Informaram } \\
\text { Cronograma } \\
\text { de Execução }\end{array}$ & $\begin{array}{c}\text { EM01, EM02, EM03, EM05, EM06, } \\
\text { EM10, EM11, EM13, EM14, EM15 }\end{array}$ & EM09, EM12 & 12 \\
\hline $\begin{array}{c}\text { Não } \\
\text { Informaram } \\
\begin{array}{l}\text { Cronograma } \\
\text { de Execução }\end{array}\end{array}$ & EM04, EM07, EM08, EM17 & EM16 & 05 \\
\hline Total & $\mathbf{1 4}$ & $\mathbf{0 3}$ & $\mathbf{1 7}$ \\
\hline
\end{tabular}

\section{QPS3 - São mencionadas as ameaças à validade e também como essas ameaças afetam os resultados e conclusões?}

\section{Empatia}

Em relação a Empatia e conforme apresentado na Tabela 17, somente 5 estudos não apresentaram ameaças à validade e 74 apresentaram. Vale ressaltar que é importante informar as ameaças para que em uma eventual replicação tenha-se noção de fatores de risco à validação e como mitigar esses fatores. Logo abaixo a Tabela 15 com os dados dos estudos: 
Tabela 15: Estudos Selecionados por Informação de Ameaças à Validade referentes ao Subtema 1 (Empatia).

\begin{tabular}{|c|c|c|c|}
\hline Ameaça & Estudos Estrangeiros & Estudos Brasileiros & $\begin{array}{c}\text { Quantitativo de } \\
\text { Estudos }\end{array}$ \\
\hline $\begin{array}{l}\text { Apresentaram } \\
\text { Ameaças à } \\
\text { Validade }\end{array}$ & $\begin{array}{l}\text { EE01, EE02, EE03, EE04, EE06, EE07, } \\
\text { EE08, EE09, EE11, EE12, EE13, EE14, } \\
\text { EE15, EE16, EE17, EE18, EE19, EE20, } \\
\text { EE21, EE22, EE23, EE24, EE25, EE26, } \\
\text { EE27, EE28, EE29, EE30, EE31, EE32, } \\
\text { EE33, EE34, EE35, EE36, EE37, EE38, } \\
\text { EE39, EE40, EE41, EE42, EE43, EE44, } \\
\text { EE45, EE46, EE47, EE48, EE49, EE50, } \\
\text { EE51, EE52, EE53, EE54, EE55, EE56, } \\
\text { EE57, EE58, EE59, EE60, EE61, EE62, } \\
\text { EE66, EE67, EE68, EE72, EE79 }\end{array}$ & $\begin{array}{l}\text { EE10, EE63, EE64, } \\
\text { EE65, EE69, EE71, } \\
\text { EE74, EE77, EE78 }\end{array}$ & 74 \\
\hline $\begin{array}{c}\text { Não } \\
\text { Apresentaram } \\
\text { Ameaças à } \\
\text { Validade }\end{array}$ & EE05, EE70 & EE73, EE75, EE76 & 5 \\
\hline Total & 67 & 12 & 79 \\
\hline
\end{tabular}

\section{Mindfulness}

Nos estudos referentes a Mindfulness, 5 não elencam as ameaças à validade descritos na Tabela 16. Um ponto importante citado pelos estudos que contemplaram essa questão é a limitação do número de pessoas presentes nos estudos, e até a sugestão de replicação para uma validação mais consistente.

Tabela 16: Estudos Selecionados por Informação de Ameaças à Validade referentes ao Subtema 2 (Mindfulness).

\begin{tabular}{|c|c|c|c|}
\hline Ameaça & Estudos Estrangeiros & Estudos Brasileiros & $\begin{array}{c}\text { Quantitativo de } \\
\text { Estudos }\end{array}$ \\
\hline $\begin{array}{c}\text { Apresentaram } \\
\text { Ameaças à } \\
\text { Validade }\end{array}$ & $\begin{array}{c}\text { EM01, EM03, EM04, EM05, } \\
\text { EM06, EM07, EM08, EM13, } \\
\text { EM14, EM15, EM17 }\end{array}$ & EM16 & 12 \\
\hline $\begin{array}{c}\text { Não } \\
\text { Apresentaram } \\
\text { Ameaças à } \\
\text { Validade }\end{array}$ & EM02, EM10, EM11 & EM09, EM12 & 5 \\
\hline Total & $\mathbf{1 4}$ & $\mathbf{3}$ & $\mathbf{1 7}$ \\
\hline
\end{tabular}

\section{QPS4 - Há algum custo para implementação?}

\section{Empatia}

Nos estudos sobre Empatia, dos 79 estudos apenas 1 estudo estrangeiro informou de forma clara o custo para implementação (EE02) no valor de US\$ 5.600,00 dólares. Destaca-se que é importante esta informação para que os gestores possam escolher a intervenção que se adeque também a sua realidade financeira.

\section{Mindfulness}

Uma tendência presenciada em ambos os Subtemas foi a ausência de detalhamentos referentes ao custo de implantação. Nenhum dos 17 estudos sobre Mindfulness apresentou valores. Acredita-se que as atividades propostas para a prática de Mindfulness sejam de baixo custo, de forma a facilitar a sua replicação e da falta de necessidade de detalhamento pelos pesquisadores/autores. 


\section{QPS5 - Qual tipo de escola o estudo é realizado?}

\section{Empatia}

Quanto aos dados analisados em relação aos estudos de Empatia e conforme a Tabela 17, 31 estudos foram em escolas públicas, 4 em escolas particulares e 44 não informaram o tipo de escola. Desse total 12 escolas são brasileiras, sendo que 8 foram em escolas públicas, 3 em escolas privadas e 1 não informou o tipo de escola. Nota-se uma tendência a não informar o tipo de escola que recebe a intervenção em contexto estrangeiro, diferentemente do contexto brasileiro.

Tabela 17: Distribuição dos Estudos Selecionados por Tipo de Escola para o Subtema 1 (Empatia).

\begin{tabular}{|c|c|c|c|}
\hline Tipo de Escola & Estudos Estrangeiros & Estudos Brasileiros & $\begin{array}{l}\text { Quantitativo de } \\
\text { Estudos }\end{array}$ \\
\hline Pública & $\begin{array}{l}\text { EE02, EE04, EE06, EE08, EE11, EE16, } \\
\text { EE17, EE19, EE23, EE25, EE29, EE34, } \\
\text { EE37, EE38, EE45, EE48, EE50, EE59, } \\
\text { EE60, EE61, EE62, EE68, EE79 }\end{array}$ & $\begin{array}{l}\text { EE10, EE63, EE64, } \\
\text { EE69, EE71, EE76, } \\
\text { EE77, EE78 }\end{array}$ & 31 \\
\hline Privada & EE66 & EE65, EE73, EE75 & 04 \\
\hline Não especificado & $\begin{array}{c}\text { EE01, EE03, EE05, EE07, EE09, EE12, } \\
\text { EE13, EE14, EE15, EE18, EE20, EE21, } \\
\text { EE22, EE24, EE26, EE27, EE28, } \\
\text { EE30, EE31, EE32, EE33, EE35, EE36, } \\
\text { EE39, EE40, EE41, EE42, EE43, EE44, } \\
\text { EE46, EE47, EE49, EE51, EE52, EE53, } \\
\text { EE54, } \\
\text { EE55, EE56, EE57, EE58, EE67, } \\
\text { EE70, EE72 }\end{array}$ & EE74 & 44 \\
\hline
\end{tabular}

\section{Mindfulness}

O ambiente escolar escolhido pela maioria dos pesquisadores para a prática de Mindfulness foi o público, com 13 dos 16 estudos. Apenas 1 dos selecionados não especificou em que tipo de escola o experimento foi aplicado como mostra a Tabela 18. Dos 3 estudos aplicados em escolas privadas, dois foram realizados em escolas brasileiras. Para os estudos estrangeiros, nota-se uma tendência de aplicação em escolas públicas.

Tabela 18: Distribuição dos Estudos Selecionados por Tipo de Escola para o Subtema 2 (Mindfulness).

\begin{tabular}{|c|c|c|c|}
\hline Tipo de Escola & Estudos Estrangeiros & Estudos Brasileiros & $\begin{array}{c}\text { Quantitativo de } \\
\text { Estudos }\end{array}$ \\
\hline \multirow{3}{*}{ Publica } & EM01, EM02, EM03, EM04, & & 13 \\
& $\begin{array}{c}\text { EM05, EM06, EM07, EM10, } \\
\text { EM11, EM13, EM14, EM15, } \\
\text { EM17 }\end{array}$ & - & 03 \\
\hline Privada & EM08 & & 01 \\
\hline Não especificado & - & EM09, EM16 & \\
\hline
\end{tabular}

\section{QPS6 - Qual o tipo de intervenção foi realizada?}

\section{Empatia}

Quanto aos dados analisados em relação aos estudos de Empatia e conforme a Tabela 19, foram feitos estudos do tipo estudo caso-controle (experimental) (32), estudo quase-experimental (23), estudo de caso (15), estudo longitudinal (6) e estudo descritivo (2). No Brasil, os tipos de intervenções realizadas são caso-controle (experimental) (1), estudo quase-experimental (6), 
estudo de caso (3) e estudo descritivo (2). Nota-se uma tendência a estudos que levem um rigor estatístico em suas evidências, com intervenções de caso-controle (experimental) no contexto estrangeiro e no contexto brasileiro com intervenção quase-experimental.

Tabela 19: Distribuição dos Estudos Selecionados por Tipo de intervenção para o Subtema 1 (Empatia).

\begin{tabular}{|c|c|c|c|}
\hline $\begin{array}{c}\text { Tipo de } \\
\text { Intervenção }\end{array}$ & Estudos Estrangeiros & Estudos Brasileiros & $\begin{array}{c}\text { Quantitativo de } \\
\text { Estudos }\end{array}$ \\
\hline $\begin{array}{l}\text { Caso-Controle } \\
\text { (Experimental) }\end{array}$ & $\begin{array}{l}\text { EE04, EE05, EE09, EE11, EE15, } \\
\text { EE21, EE22, EE24, EE27, EE30, } \\
\text { EE31, EE33, EE34, EE35, EE36, } \\
\text { EE37, EE38, EE40, EE41, EE45, } \\
\text { EE46, EE50, EE52, EE53, EE55, } \\
\text { EE57, EE60, EE62, EE66, EE67, } \\
\text { EE70, EE79 }\end{array}$ & EE69 & - somicos \\
\hline Quase-experimental & $\begin{array}{l}\text { EE02, EE08, EE12, EE14, EE17, } \\
\text { EE19, EE25, EE26, EE31, EE29, } \\
\text { EE43, EE44, EE47, EE49, EE51, } \\
\text { EE56, EE72 }\end{array}$ & $\begin{array}{l}\text { EE10, EE64, EE71, } \\
\text { EE74, EE76, EE77 }\end{array}$ & 23 \\
\hline Longitudinal & $\begin{array}{c}\text { EE03, EE20, EE28, EE39, EE58, } \\
\text { EE59 }\end{array}$ & - & 06 \\
\hline Estudo de Caso & $\begin{array}{l}\text { EE01, EE06, EE07, EE13, EE68 } \\
\text { EE16, EE18, EE23, EE32, EE42, } \\
\text { EE48, EE54, EE61 }\end{array}$ & EE63, EE65, EE75 & 16 \\
\hline Estudo Descritivo & - & EE73, EE78 & 02 \\
\hline
\end{tabular}

\section{Mindfulness}

Quanto a Mindfulness os estudos se concentraram em caso-controle (experimental). Acredita-se que pelo caráter de sua aplicação.

\section{QPS7 - Qual o tipo de público do estudo?}

\section{Empatia}

Quanto aos dados analisados em relação aos estudos de Empatia, foram direcionados para Alunos (60); Alunos e Professores (2); Professores (13); Pais (1); Alunos e Pais (1); Alunos, Pais e Funcionários (1); e Alunos, Pais e Professores (1). No contexto brasileiro foram direcionados para Alunos (10) e Professores (2). Vale ressaltar, que há estudos que contemplam mais de um tipo de público ao mesmo tempo como mostrado na Tabela 22.

Tabela 22: Distribuição dos Estudos Selecionados por Público do estudo - Subtema 1 (Empatia).

\begin{tabular}{|c|c|c|c|}
\hline Público & Estudos Estrangeiros & $\begin{array}{c}\text { Estudos } \\
\text { Brasileiros }\end{array}$ & $\begin{array}{c}\text { Quantitativo de } \\
\text { Estudos }\end{array}$ \\
\hline & EE01, EE02, EE03, EE04, EE06, EE07, & & \\
& EE08, EE11, EE12, EE13 EE15, EE16, EE17, \\
EE18, EE20, EE22, EE23, EE24, EE25, & EE10, EE63, & EE64, EE65, \\
& EE26, EE28, EE29, EE30, EE31, EE32, & EE69, EE71, & 60 \\
& EE33, EE34, EE35, EE38, EE39, EE40, & EE75, EE76, & \\
& EE41, EE42, EE44, EE45, EE50, EE52, & EE77, EE78 & \\
& EE53, EE54, EE55, EE56, EE57, EE58, & & 02 \\
\hline Alunos e & EE59, EE60, EE66, EE67, EE68, EE72, EE79 & & 13 \\
\hline Professores & EE27, EE62 & & 01 \\
\hline Professores & EE05, EE19, EE21, EE36, EE37, EE47, & EE73, EE74 & 01 \\
\hline Pais & EE48, EE49, EE51, EE61, EE70 & - & - \\
\hline Alunos e Pais & EE09 & EE46 & \\
\hline
\end{tabular}




\begin{tabular}{|c|c|c|c|}
\hline $\begin{array}{c}\text { Funcionários, } \\
\text { Pais, Professores }\end{array}$ & EE43 & - & 01 \\
\hline $\begin{array}{c}\text { Alunos, Pais e } \\
\text { Professores }\end{array}$ & EE14 & - & 01 \\
\hline
\end{tabular}

\section{Mindfulness}

A aplicação de Mindfulness se assemelha a da Empatia, onde grande parte dos estudos (12) se concentram no aluno. Três estudos focam tanto no Professores quanto no Alunos e somente um foi direcionado aos Professores e aos Alunos e Funcionários. A aplicação de Mindfulness direcionada apenas para os funcionários não foi encontrada, como mostra a Tabela 23.

Tabela 23: Distribuição dos Estudos Selecionados por Público do estudo - Subtema 2 (Mindfulness).

\begin{tabular}{|c|c|c|c|}
\hline Público & Estudos Estrangeiros & Estudos Brasileiros & Quantitativo de Estudos \\
\hline \multirow{2}{*}{ Alunos } & EM03, EM04, EM06, & & 12 \\
& $\begin{array}{c}\text { EM07, EM08, EM11, } \\
\text { EM13, EM15, RM17 }\end{array}$ & EM09, EM12, EM16 & 03 \\
\hline Alunos e Professores & EM01, EM02, EM14 & - & 01 \\
\hline Alunos e Funcionários & EM05 & - & 01 \\
\hline Professores & EM10 & - & 0 \\
\hline Funcionários & - & - & \\
\hline
\end{tabular}

\section{QPS8 - Qual a técnica/método estatístico usado para avaliar a evidência?}

\section{Empatia}

Quanto aos dados analisados em relação aos estudos de Empatia, 62 estudos utilizam algum tipo de técnica/método e 17 não utilizam. Das técnicas utilizadas como mostrada na Tabela 24 destacam-se média (48), desvio padrão (48), teste t (25), ANOVA (17). Vale ressaltar que vários estudos utilizam mais de uma técnica/método estatístico para avaliar suas evidências, assim como testes (EE74, EE76, EE77) para medir Empatia antes e após o experimento. A Tabela abaixo divide os estudos no contexto nacional e estrangeiro.

Tabela 24: Distribuição dos Estudos Selecionados por Técnica/Método Estatístico - Subtema 1 (Empatia).

\begin{tabular}{|c|c|c|c|}
\hline $\begin{array}{l}\text { Técnicas/Métodos } \\
\text { Estatísticos }\end{array}$ & Estudos Estrangeiros & $\begin{array}{c}\text { Estudos } \\
\text { Brasileiros }\end{array}$ & $\begin{array}{c}\text { Quantitativo de } \\
\text { Estudos }\end{array}$ \\
\hline Média & $\begin{array}{l}\text { EE02, EE03, EE05, EE08, EE11, EE12, } \\
\text { EE14, EE15, EE17, EE19, EE20, EE21, } \\
\text { EE22, EE25, EE26, EE27, EE29, EE30, } \\
\text { EE31, EE33, EE35, EE36, EE37, EE38, } \\
\text { EE39, EE43, EE44, EE45, EE46, EE47, } \\
\text { EE49, EE51, EE52, EE53, EE55, EE56, } \\
\text { EE57, EE58, EE59, EE60, EE62, EE70, } \\
\text { EE72 }\end{array}$ & $\begin{array}{l}\text { EE64, EE69, } \\
\text { EE71, EE76, } \\
\text { EE77 }\end{array}$ & 48 \\
\hline Desvio Padrão & $\begin{array}{l}\text { EE02, EE03, EE05, EE11, EE12, EE14, } \\
\text { EE17, EE19, EE20, EE21, EE22, EE25, } \\
\text { EE26, EE27, EE29, EE30, EE31, EE33, } \\
\text { EE35, EE36, EE37, EE38, EE39, EE41, } \\
\text { EE43, EE44, EE45, EE46, EE47, EE49, } \\
\text { EE51, EE52, EE53, EE55, EE56, EE57, } \\
\text { EE58, EE59, EE60, EE62, EE67, EE70, } \\
\text { EE72, EE73 }\end{array}$ & $\begin{array}{l}\text { EE64, EE69, } \\
\text { EE71, EE76, } \\
\text { EE77 }\end{array}$ & 48 \\
\hline Teste $\mathrm{t}$ & $\begin{array}{c}\text { EE04, EE05, EE09, EE19, EE25, } \\
\text { EE26, EE34, EE35, EE36, EE37, } \\
\text { EE38, EE39, EE41, EE44, EE47, } \\
\text { EE51, EE59, EE60, EE62, EE67, EE70 }\end{array}$ & $\begin{array}{l}\text { EE64, EE74, } \\
\text { EE78, EE79 }\end{array}$ & 25 \\
\hline ANOVA & EE02, EE14, EE17, EE22, EE30, & EE77 & 17 \\
\hline
\end{tabular}




\begin{tabular}{|c|c|c|c|}
\hline & $\begin{array}{c}\text { EE31, EE33, EE35, EE36, EE40, } \\
\text { EE45, EE46, EE50, EE57, EE58, } \\
\text { EE72 }\end{array}$ & & \\
\hline Tamanho do Efeito & $\begin{array}{l}\text { EE20, EE22, EE31, EE36, EE49, } \\
\text { EE56, EE62 }\end{array}$ & - & 07 \\
\hline MANOVA & EE02, EE30, EE31, EE36 & - & 04 \\
\hline MANCOVA & EE25, EE30, EE72 & - & 03 \\
\hline Mínimo & EE49, EE60 & EE69 & 03 \\
\hline Máximo & EE49, EE60 & EE69 & 03 \\
\hline Mediana & EE52 & EE69, EE71 & 03 \\
\hline $\begin{array}{l}\text { Teste U de Mann- } \\
\text { Witney }\end{array}$ & EE21, EE52 & EE69, EE71 & 04 \\
\hline Teste Exato de Fisher & - & EE10 & 01 \\
\hline $\begin{array}{l}\text { Qui-Quadrado de } \\
\text { Pearson }\end{array}$ & - & EE10 & 01 \\
\hline Alfa de Cronbach & EE11, EE43 & - & 02 \\
\hline $\begin{array}{l}\text { Regressão linear } \\
\text { múltipla }\end{array}$ & EE11 & - & 01 \\
\hline Spearman & EE21 & - & 01 \\
\hline Análise de Variância & EE41 & - & 01 \\
\hline $\begin{array}{l}\text { Média de Variância } \\
\text { Extraída (AVE) }\end{array}$ & EE51 & - & 01 \\
\hline Correlações de Pearson & EE57 & - & 01 \\
\hline $\begin{array}{l}\text { Coeficientes de } \\
\text { Correlação Intracluster } \\
\text { (ICC) }\end{array}$ & EE58 & - & 01 \\
\hline $\begin{array}{l}\text { Confiabilidade } \\
\text { Composta }\end{array}$ & EE51 & - & 01 \\
\hline $\begin{array}{l}\text { Modelagem Estatística } \\
\text { de Formas }\end{array}$ & EE68 & - & 01 \\
\hline Wilcoxon & - & EE71 & 01 \\
\hline Kolmogorov-Smirnov & - & EE74 & 01 \\
\hline $\begin{array}{l}\text { Regressão de Poisson } \\
\text { com Efeito Aleatório }\end{array}$ & - & EE76 & 01 \\
\hline
\end{tabular}

\section{Mindfulness}

Não foi identificada nenhuma padronização no que se trata a métodos estatísticos usados para avaliar as evidências. Muitos estudos apresentaram diversos questionários e avaliações. Dos identificados podemos elencar Cronbach (EM06), Pearson (EM07) e Cohen (EM14).

\section{QPS9 - Sobre qual área/subárea a intervenção é realizada?}

\section{Empatia}

Quanto aos dados analisados em relação aos estudos de Empatia e conforme a Tabela 25, as intervenções são na área/subárea Socioemocional - Intervenções na Educação Básica (60), Socioemocional - Formação de Professores (12), História - Empatia Histórica (4), Socioemocional - Validação de Ferramenta (2) e Socioemocional - Intervenções na família (1).

Tabela 25: Distribuição dos Estudos Selecionados por Área/Subárea para o Subtema 1 (Empatia).

\begin{tabular}{|c|c|c|c|}
\hline Área/Subárea & Estudos Estrangeiros & Estudos Brasileiros & $\begin{array}{c}\text { Quantitativo de } \\
\text { Estudos }\end{array}$ \\
\hline & EE02, EE03, EE04, EE06, EE07, & & \\
Socioemocional - & EE11, EE12, EE13, EE14, EE15, & & 60 \\
Intervenções na & EE16, EE17, EE18, EE20, EE22, & EE10, EE64, EE69, EE71, & \\
Educação Básica & EE23, EE24, EE25, EE26, EE27, & EE75, EE76, EE77, EE78 & \\
& EE28, EE30, EE31, EE32, EE33, & & \\
& EE34, EE35, EE38, EE39, EE40, & & \\
\hline
\end{tabular}




\begin{tabular}{|c|c|c|c|}
\hline & $\begin{array}{c}\text { EE41, EE42, EE43, EE44, EE45, } \\
\text { EE46, EE50, EE51, EE52, EE53, } \\
\text { EE54, EE55, EE56, EE57, EE58, } \\
\text { EE59, EE60, EE62, EE66, EE67, } \\
\text { EE68, EE79 }\end{array}$ & & \\
\hline $\begin{array}{c}\text { Socioemocional - } \\
\text { Formação de } \\
\text { Professores }\end{array}$ & $\begin{array}{c}\text { EE05, EE19, EE21, EE36, EE37, } \\
\text { EE47, EE48, EE49, EE61, EE70 }\end{array}$ & EE73, EE74 & 12 \\
\hline $\begin{array}{c}\text { História - Empatia } \\
\text { Histórica }\end{array}$ & EE01, EE08 & EE63, EE65 & 04 \\
\hline $\begin{array}{c}\text { Socioemocional - } \\
\text { Validação de } \\
\text { Ferramenta }\end{array}$ & EE29, EE72 & & 02 \\
\hline $\begin{array}{c}\text { Socioemocional - } \\
\text { Intervenções na } \\
\text { família }\end{array}$ & EE09 & & 01 \\
\hline
\end{tabular}

\section{Mindfulness}

Pelo caráter das atividades, as aplicações de Mindfulness se distanciaram das disciplinas e sua intervenção foi realizada em momentos extra-classe ou intervalos.

\section{QPS10 - Qual a escolaridade dos participantes?}

\section{Empatia}

Quanto aos dados analisados em relação aos estudos de Empatia, somente 46 estudos informam com clareza a escolaridade dos participantes e 33 não informam.

Desses 46, destacam-se o Ensino Superior (11), Fundamental $6^{\circ}$ ano (11), Fundamental $5^{\circ}$ ano (11). Vale ressaltar que pela grande quantidade de estudos de nível superior, não foi incluído os estudos que tinham como foco os alunos, mas incluídos os estudos que tinham como foco os professores, pois são em menor quantidade.

Abaixo, é apresentado na Tabela 26 informações referentes a quantidade, identificação e escolaridade dos participantes desses estudos. 
Tabela 26: Distribuição dos Estudos Selecionados por Escolaridade dos Participantes para o Subtema 1 (Empatia).

\begin{tabular}{|c|c|c|c|}
\hline Escolaridade & Estudos Estrangeiros & Estudos Brasileiros & $\begin{array}{c}\text { Quantitativo de } \\
\text { Estudos } \\
\end{array}$ \\
\hline Creche & EE14, EE16 & EE75 & 03 \\
\hline Pré-escola & EE04, EE42, EE46 & - & 03 \\
\hline Fund. $1^{\circ}$ ano & EE01, EE68 & EE10, EE69 & 04 \\
\hline Fund. $2^{\circ}$ ano & EE01, EE22, EE24, EE59, EE68 & EE71 & 06 \\
\hline Fund. $3^{\circ}$ ano & $\begin{array}{c}\text { EE01, EE23, EE56, EE59, EE60, } \\
\text { EE68, EE72 }\end{array}$ & - & 07 \\
\hline Fund. $4^{\circ}$ ano & $\begin{array}{c}\text { EE01, EE02, EE34, EE41, EE56, } \\
\text { EE59, EE62, EE72 }\end{array}$ & - & 08 \\
\hline Fund. $5^{\circ}$ ano & $\begin{array}{c}\text { EE02, EE07, EE23, EE33, EE35, } \\
\text { EE41, EE54, EE56, EE58, EE59, } \\
\text { EE62 }\end{array}$ & - & 11 \\
\hline Fund. $6^{\circ}$ ano & $\begin{array}{l}\text { EE02, EE07, EE20, EE29, EE38, } \\
\text { EE54, EE56, EE59, EE62, EE79 }\end{array}$ & EE73, EE76 & 12 \\
\hline Fund. $7^{\circ}$ ano & $\begin{array}{l}\text { EE02, EE11, EE29, EE34, EE38, } \\
\text { EE39, EE54, EE56, EE67, EE79 }\end{array}$ & EE73 & 11 \\
\hline Fund. $8^{\circ}$ ano & $\begin{array}{l}\text { EE11, EE29, EE34, EE35, EE39, } \\
\text { EE54, EE56, EE79 }\end{array}$ & EE73 & 09 \\
\hline Fund. $9^{\circ}$ ano & $\begin{array}{c}\text { EE03, EE11, EE29, EE34, EE55, } \\
\text { EE56 }\end{array}$ & EE63, EE73, EE78 & 09 \\
\hline Médio $1^{\circ}$ ano & $\begin{array}{c}\text { EE03, EE08, EE11, EE17, EE29, } \\
\text { EE52, EE53, EE56 }\end{array}$ & EE65, EE73, EE77 & 11 \\
\hline Médio $2^{\circ}$ ano & $\begin{array}{c}\text { EE03, EE08, EE11, EE17, EE29, } \\
\text { EE52, EE53, EE56 }\end{array}$ & EE65, EE73 & 10 \\
\hline Médio $3^{\circ}$ ano & $\begin{array}{l}\text { EE03, EE17, EE29, EE52, EE53, } \\
\text { EE56 }\end{array}$ & EE65, EE73 & 08 \\
\hline Ensino Superior & $\begin{array}{l}\text { EE05, EE19, EE21, EE36, EE37, } \\
\text { EE47, EE48, EE49, EE61, EE70 }\end{array}$ & EE73, EE74 & 12 \\
\hline
\end{tabular}

\section{Mindfulness}

Os estudos selecionados referentes a Mindfulness se concentram em Ensino Superior (7) Fundamental do $1^{\circ}$ ao $4^{\circ}$ ano (4) e Fundamental do $7^{\circ}$ ao $9^{\circ}$ ano (4) assim apresentados na Tabela 27. Somente dois estudos foram aplicados na pré-escola como apresentados.

Vale pontuar que para a sua aplicação no Brasil os pesquisadores se limitaram ao $7^{\circ}$ ano do ensino Fundamental e diferentemente da aplicação de Empatia, o $5^{\circ}$ e $6^{\circ}$ ano do ensino Fundamental e o ensino Médio.

Tabela 27: Distribuição dos Estudos Selecionados por Escolaridade dos participantes - Subtema 2 (Mindfulness).

\begin{tabular}{|c|c|c|c|}
\hline Escolaridade & Estudos Estrangeiros & Estudos Brasileiros & $\begin{array}{c}\text { Quantitativo de } \\
\text { Estudos }\end{array}$ \\
\hline Pre-escola & EM10, EM13 & - & 02 \\
\hline Fund. $1^{\circ}$ ano & EM10 & - & 01 \\
\hline Fund. $2^{\circ}$ ano & EM01, EM02, EM10, EM15 & - & 04 \\
\hline Fund. $3^{\circ}$ ano & EM01, EM02, EM10, EM15 & - & 04 \\
\hline Fund. $4^{\circ}$ ano & EM02, EM10 & - & 02 \\
\hline Fund. $7^{\circ}$ ano & EM14 & EM09, EM12, EM16 & 01 \\
\hline Fund. $8^{\circ}$ ano & EM14 & - & 01 \\
\hline Fund. $9^{\circ}$ ano & EM14 & - & 07 \\
\hline Ensino Superior & $\begin{array}{c}\text { EM03, EM04, EM05, EM07, } \\
\text { EM08, EM11, EM17 }\end{array}$ & - & 04 \\
\hline
\end{tabular}




\section{QPS11 - Há o uso de tecnologias educacionais digitais ou educacionais analógicas?}

\section{Empatia}

Quanto aos dados analisados em relação aos estudos de Empatia, os principais tipos de tecnologia empregados são Jogos, Programa Socioemocional, Literatura e Didática. Dentro dessas tecnologias há estudos que trabalham com:

Jogos: jogos digitais (EE01, EE13, EE16), brincadeiras infantis (EE34, EE75), jogos cooperativos (EE14), etc.

Programa Socioemocional: pintura (EE74), música (EE63), vídeos (EE20, EE30, EE31), produção de curta-metragem (EE54), dramatização (EE74, EE35, EE40), co-design (EE06, EE18), etc.

Literatura: leitura de livros (EE66, EE77), histórias digitais (EE16, EE42), biblioterapia (EE26), etc.

Abaixo, é apresentado na Tabela 28 informações referentes a quantidade, identificação e tecnologia educacional digital ou educacional analógica desses estudos. Vale ressaltar que consideramos que tecnologias educacionais digitais são aquelas que permitem a transformação de instrumentos educacionais (imagem, texto, jogos, sons, etc.) do mundo real em informação digital, que é vista através de dispositivos digitais (computadores, tablet, etc) e que tem como objetivo o apoio no processo de ensino-aprendizagem. Tecnologias educacionais analógicas não passaram pelo processo de digitalização desses instrumentos, não é necessário o uso de dispositivos digitais e também tem o mesmo objetivo das tecnologias educacionais digitais, mas ela também pode ser transformada em tecnologia educacional digital.

Tabela 28: Distribuição dos Estudos Selecionados por Tecnologia Educacional Digital ou Analógica para o Subtema 1 (Empatia).

\begin{tabular}{|c|c|c|c|c|}
\hline $\begin{array}{l}\text { Tecnologia } \\
\text { Educacional } \\
\text { Digital }\end{array}$ & $\begin{array}{l}\text { Tecnologia } \\
\text { Educacional } \\
\text { Analógica }\end{array}$ & Estudos Estrangeiros & $\begin{array}{c}\text { Estudos } \\
\text { Brasileiros }\end{array}$ & $\begin{array}{c}\text { Quantitativ } \\
\text { o de } \\
\text { Estudos }\end{array}$ \\
\hline $\begin{array}{l}\text { Realidade } \\
\text { Aumentada }\end{array}$ & - & EE24 & - & 01 \\
\hline- & $\begin{array}{c}\text { Programa } \\
\text { socioemocional }\end{array}$ & $\begin{array}{l}\text { EE02, EE05, EE09, EE12, } \\
\text { EE14, EE19, EE25, EE32, } \\
\text { EE36, EE38, EE41, EE46, } \\
\text { EE49, EE53, EE59, EE60, } \\
\text { EE62 }\end{array}$ & $\begin{array}{l}\text { EE10, EE69, } \\
\text { EE71, EE74, } \\
\text { EE78 }\end{array}$ & 21 \\
\hline Realidade Virtual & - & EE21, EE39, EE43 & - & 03 \\
\hline- & Didática & $\begin{array}{l}\text { EE03, EE08, EE20, EE33, } \\
\text { EE51, EE55, EE57, EE58, } \\
\text { EE67, EE79 }\end{array}$ & - & 10 \\
\hline- & Desenho & EE04, EE14, EE22 & - & 03 \\
\hline- & Co-design & EE06, EE17, EE18 & - & 03 \\
\hline- & Yoga & EE07, EE23 & - & 02 \\
\hline- & Mindfulness & EE11, EE23 & - & 02 \\
\hline- & Figuras plásticas & EE16 & - & 01 \\
\hline- & Pintura & EE16 & EE74 & 02 \\
\hline Vídeos & - & $\begin{array}{l}\text { EE20, EE30, EE31, EE35, } \\
\text { EE51, EE56, EE61 }\end{array}$ & EE76 & 08 \\
\hline- & Dramatização & $\begin{array}{c}\text { EE03, EE14, EE25, EE30, } \\
\text { EE31, EE35, EE40, EE51, } \\
\text { EE61 }\end{array}$ & $\begin{array}{l}\text { EE65, EE74, } \\
\text { EE76 }\end{array}$ & 12 \\
\hline Música & - & EE40 & EE63 & 02 \\
\hline Imagens & - & EE40, EE57 & - & 02 \\
\hline- & Brainstorming & EE30, EE31 & - & 02 \\
\hline
\end{tabular}




\begin{tabular}{|c|c|c|c|c|}
\hline- & Debates & EE03, EE22, EE30, EE31 & - & 04 \\
\hline- & Fórum & EE25 & - & 01 \\
\hline Fotos & - & EE25 & - & 01 \\
\hline Robótica & - & EE68 & EE74 & 01 \\
\hline $\begin{array}{c}\text { Produção de } \\
\text { curta-metragem }\end{array}$ & Corte e colagem & EE54 & - & 01 \\
\hline & Projeto Artistico & EE25 & EE64 & 02 \\
\hline Aplicativo Móvel & & EE28 & - & 01 \\
\hline & Curso & EE37, EE70 & - & 02 \\
\hline $\begin{array}{c}\text { Ambiente de } \\
\text { aprendizagem } \\
\text { virtual }\end{array}$ & Workshop & EE47 & & 01 \\
\hline colaborativo & - & EE15 & - & 01 \\
\hline Filmes & Técnicas cognitivo- & EE25 & EE74 & 02 \\
\hline- & Atividade de Grupo & EE03, EE25, EE34, EE74 & & 04 \\
\hline- & Diário de Aula & & EE73 & 01 \\
\hline- & $\begin{array}{c}\text { Entrevista } \\
\text { semiestruturada }\end{array}$ & EE16 & EE73 & 02 \\
\hline- & moderamento positivo & - & EE14, EE76 & 02 \\
\hline- & feedback & - & EE76 & 01 \\
\hline
\end{tabular}

Também foram encontrados estudos que possuíam tecnologias educacionais que foram usadas em seu formato digital e em outros estudos em seu formato analógico. Para uma melhor compreensão, esses estudos foram separados e colocados na Tabela 29, logo abaixo.

Tabela 29: Distribuição dos Estudos Selecionados que Usaram Tecnologia Educacional em seu Formato Digital / Analógica para o Subtema 1 (Empatia).

\begin{tabular}{|c|c|c|c|c|c|c|}
\hline \multirow{2}{*}{$\begin{array}{c}\text { Tipo de } \\
\text { Tecnologia }\end{array}$} & \multicolumn{3}{|c|}{ Jogos } & \multicolumn{3}{|c|}{ Literatura } \\
\hline & $\begin{array}{c}\text { Estudos } \\
\text { Estrangeiros }\end{array}$ & $\begin{array}{c}\text { Estudos } \\
\text { Brasileiros }\end{array}$ & $\begin{array}{l}\text { Quantidade } \\
\text { de Estudos }\end{array}$ & $\begin{array}{c}\text { Estudos } \\
\text { Estrangeiros }\end{array}$ & $\begin{array}{c}\text { Estudos } \\
\text { Brasileiros }\end{array}$ & $\begin{array}{c}\text { Quantida } \\
\text { de de } \\
\text { Estudos }\end{array}$ \\
\hline $\begin{array}{c}\text { Educacional } \\
\text { Digital }\end{array}$ & $\begin{array}{l}\text { EE01, EE13, } \\
\text { EE16, EE26, } \\
\text { EE29, EE44, } \\
\text { EE45, EE50, } \\
\text { EE52, EE72 }\end{array}$ & - & 10 & $\begin{array}{l}\text { EE14, EE16, } \\
\text { EE25, EE27, } \\
\text { EE42, EE48 }\end{array}$ & - & 06 \\
\hline $\begin{array}{l}\text { Educacional } \\
\text { Analógico }\end{array}$ & EE03, EE14 & EE75 & 3 & EE16, EE66 & EE74, EE77 & 04 \\
\hline
\end{tabular}

\section{Mindfulness}

A aplicação de Mindfulness relatada pelos estudos segue protocolos desenvolvidos pelos professores/pesquisadores como: Cognitive and Affective Mindfulness Scale - Revised (CAMSR), Five Factor Mindfulness Questionnaire (FFMQ), Freiburg Mindfulness Inventory (FMI), Kentucky Inventory of Mindfulness Skills (KIMS), Mindful Attention Awareness Scale (MAAS) entre outros. Nos achados, infere-se a ausência de padrões nas aplicações, mesmo com diversos protocolos já implementados. Os estudos não apresentam nenhuma tecnologia educacional clara, nem digital, nem analógica a não ser a utilização de um aplicativo para o auxílio da meditação (EM05). 


\section{QPP: Quais evidências existem que relacionam o uso de tecnologias educacionais para o desenvolvimento de habilidades socioemocionais no ensino básico brasileiro?}

A partir das questões secundárias realizadas neste MSL, é possível inferir que foram encontradas tecnologias que são utilizadas como apoio ao processo de ensino-aprendizagem no desenvolvimento de habilidades socioemocionais (Empatia e Mindfulness), levando os participantes a entender perspectivas diferentes e desenvolver a atenção plena.

Em relação a Empatia, algumas tecnologias, tais como as histórias digitais, poderiam ser usadas de maneira desplugada (HQs/Gibis) ou no formato de arquivo (pdf, etc.) em contextos emergenciais, por exemplo, a pandemia que estamos vivendo, onde algumas pessoas não têm acesso a tecnologia (computador, internet, etc.) e que, há bastante espaço para novas tecnologias como apoio ao processo de ensino-aprendizagem e entendimento de conceitos de Empatia, por exemplo, o Pensamento Computacional que pode ser um método promissor. Além disso, há o uso de métodos estatísticos em grande parte dos estudos, podendo assim medir a evolução do desenvolvimento da Empatia.

Em relação a Mindfulness, apenas um estudo (EM05) utilizou tecnologia para o auxílio da meditação, sendo que a tecnologia digital/analógica poderia ser um instrumento para facilitar o estado de atenção plena buscado pela prática de Mindfulness.

\section{Discussão}

Devido à pequena quantidade de estudos sobre Mindfulness no ensino básico, foi necessário a ampliação do escopo de pesquisa, incluindo trabalhos que visavam o ensino superior e que tivessem como foco qualquer público-alvo que participe da vida escolar (alunos, professores, funcionários, etc.), assim totalizando 17 trabalhos no total.

Por causa dessa ampliação de escopo, o Subtema Empatia também expandiu seu escopo para trabalhos que visavam o ensino superior, mas devido a alta quantidade encontrada foi necessário aumentar o escopo para trabalhos de ensino superior que tivessem como público-alvo os professores ou professores em formação.

Isso demonstra um alto interesse no Subtema 1 (Empatia) e uma escassez de trabalhos referentes ao Subtema 2 (Mindfulness), mostrando assim que o desenvolvimento da Empatia é um tema altamente relevante para a comunidade científica com muitos trabalhos sobre o tema e que o ensino de Mindfulness nas escolas é uma área de pesquisa ainda a ser explorada, sendo que a maioria dos estudos encontrados, referentes aos dois Subtemas, são publicados em periódicos tanto em caso brasileiro como em caso estrangeiro.

Em relação ao panorama nos estados brasileiros, notou-se nos estudos em território brasileiro, tanto para Mindfulness como para Empatia, uma concentração de estudos realizados nas regiões sul e sudeste, em detrimento das outras regiões brasileiras que também poderiam se beneficiar de trabalhos de desenvolvimento de habilidades socioemocionais. Apenas 15 estudos (12 de Empatia e 3 Mindfulness) foram encontrados e se considerarmos o total de nossa população estudantil (47,8 milhões) divulgados pelo Censo Escolar de 2019 (Alfano, 2020), ainda há um grande número de estudantes que podem participar de estudos que objetivam desenvolver habilidades socioemocionais.

Ao expandir o panorama para os países, é possível notar que o Brasil (12 estudos sobre Empatia) está bem posicionado ( $2^{\circ}$ colocado) em relação a quantidade de estudos relacionados ao Subtema 1 (Empatia), ao compararmos com os Estados Unidos (14 estudos sobre Empatia), o qual é o país com mais estudos encontrados sobre o Subtema 1 (Empatia), porém ao analisarmos o Subtema 2 (Mindfulness) isso não se repete, dando-se destaque para Portugal com 5 estudos 
desenvolvidos no país. Logo, mesmo a nível estrangeiro, ainda são necessárias mais intervenções que promovam o desenvolvimento de habilidades socioemocionais no ensino básico para os dois Subtemas.

Já existem países como a Dinamarca, que possui um sistema escolar que incorpora o ensino de habilidades socioemocionais a estudantes dos 6 aos 16 anos (Pensar Contemporâneo, 2019) e isso pode ter um reflexo no Índice Mundial de Felicidade 2019 (Ruic, 2019), no qual a Dinamarca está em segundo no lugar nesse índice, atrás apenas da Finlândia. Porém, nenhum estudo realizado nesse país foi encontrado em nossa busca.

Apenas um estudo (EE02), levando em conta os dois Subtemas, informou o custo para sua implementação, porém 79 estudos (67 estudos sobre Empatia e 12 sobre Mindfulness) informaram o cronograma de execução do estudo e 86 estudos (74 estudos sobre Empatia e 12 sobre Mindfulness) informaram ameaças a validade.

Essas três informações (custo, cronograma e ameaças a validade) estão intimamente ligadas e são muito importantes para os gestores públicos, já que as escolas brasileiras carecem de recursos financeiros (Santos, Nunes \& Romero, 2019). É possível que quanto maior o cronograma de execução do estudo maior será seu custo e dependendo dos recursos empregados (tecnologia, recursos humanos, etc.), torna-se inviável a replicação do estudo pretendido. Além disso, estudos possuem ameaças a validade, as quais para serem resolvidas ou mitigadas podem necessitar de um cronograma maior ou de ajustes que possivelmente podem encarecer ainda mais seu custo.

Houveram 44 estudos (31 sobre Empatia e 13 sobre Mindfulness) que foram realizados em escolas públicas e 7 estudos (4 sobre Empatia e 3 sobre Mindfulness) realizados em escolas particulares, porém 45 estudos (44 sobre Empatia e 3 sobre Mindfulness) não informaram o tipo de instituição na qual foi realizada o experimento. Ao não relatar o tipo de instituição e nem o custo do estudo, torna-se difícil traçar um paralelo sobre quanto cada tipo de instituição gasta em média para realizar esses estudos. Esse cálculo, torna-se ainda mais importante na esfera pública que geralmente lida com recursos mais escassos e que precisam ser bem aproveitados para obter o maior retorno possível no investimento feito.

Assim, há uma diversidade de tipos de intervenções encontradas nos estudos, como casocontrole (experimental) (32 sobre Empatia e 17 sobre Mindfulness), quase-experimental (23 sobre Empatia), longitudinal (06 sobre Empatia), estudo de caso (16 sobre Empatia) e descritivo (02 sobre Empatia). Cada um desses tipos de intervenção possuem características próprias e depende do estudo proposto, porém levando em conta o tempo para conclusão da intervenção e evidências de resultados dessas intervenções, as intervenções do tipo caso-controle (experimental) e quaseexperimental são as que demonstram ter um retorno sobre o investimento feito de forma mais palpável, já que utilizam questionários para avaliar as habilidades socioemocionais (Empatia / Mindfulness) antes e após as intervenções, buscando um valor quantitativo para o aumento dessas habilidades no indivíduo, por meio de técnicas/métodos estatísticos aplicados aos questionários, verificando se os dados da amostra fornecem evidência suficiente para que se possa aceitar como verdadeira a hipótese de pesquisa, precavendo-se, com certa segurança, de que as diferenças observadas nos dados não são meramente casuais.

Do total, 59 estudos (46 estudos sobre Empatia e 13 estudos sobre Mindfulness) informam a qual série do ensino básico se destina o estudo, sendo que para o ensino superior esse dado não é relevante, porém quando se trata do ensino básico, esse dado pode melhor direcionar a qual público o estudo foi aplicado e se houver a alteração desse público em uma replicação, quais são as mudanças que precisam ser feitas para contemplar um novo público-alvo, já que levando em conta o contexto brasileiro, quase 4 em cada 10 jovens ainda não concluíram o ensino médio até os 19 anos (Oliveira, 2018). Logo, é possível encontrar alunos que estão em séries que não 
condizem com a sua idade e por causa disso, possíveis ajustes podem ser feitos ao replicar um experimento em contexto brasileiro.

Em relação a Empatia, os estudos concentraram-se nos "Alunos" (60 estudos) e nos "Professores" (13 estudos) e em relação a Mindfulness, os estudos concentraram-se nos "Alunos" (12 estudos) e em "Alunos e Professores" (03 estudos). Esse achado revela que há espaço para mais pesquisas envolvendo outros participantes da vida escolar, como os pais e os funcionários da escola, já que não só o estudante, mas também as pessoas que convivem com ele podem se beneficiar de estudos cujo objetivo é o desenvolvimento de habilidades socioemocionais (Warren \& Lessner, 2014; Garaigordobil, 2012). Também é necessária uma integração ao currículo para o desenvolvimento das habilidades socioemocionais, já que a única disciplina apontada que foi usada no estudo foi a disciplina de História. Não integrar o desenvolvimento de habilidades socioemocionais as disciplinas, torna ainda mais difícil a realização de intervenções, pois assim essas habilidades são tratadas como "uma matéria a mais" e no caso brasileiro torna o calendário ainda mais apertado, já que as escolas precisam cumprir duzentos dias letivos (Senado Federal, 1996).

Em relação a Empatia, há o uso de tecnologias educacionais digitais/analógicas para apoiar o processo de desenvolvimento de habilidades socioemocionais como Empatia (Tabela 30 e Tabela 31). Quanto ao tipo de tecnologia, os resultados apontaram para maior quantidade de estudos envolvendo a utilização de Jogos que se apresentaram tanto em formato educacional analógico quanto em formato educacional digital. Tal resultado pode ser explicado pelo fato de que este instrumento possui um conjunto variado de estímulos sensoriais e de atividades, ao mesmo tempo em que trabalham o lúdico (Cordazzo \& Vieira, 2007), tornando o processo de desenvolvimento de habilidades socioemocionais mais participativo.

Em relação a Mindfulness, observa-se uma falta do uso de tecnologias educacionais digitais/analógicas utilizadas. Somente um estudo (EM05) utilizou um aplicativo para meditação, enquanto os outros estudos não apresentam nenhuma informação sobre tecnologias educacionais digitais/analógicas utilizadas, sendo que os estudos feitos poderiam se beneficiar ao incorporar tecnologias educacionais digitais/analógicas a sua intervenção.

\section{$5 \quad$ Ameaças à Validade}

Durante o desenvolvimento desta pesquisa, alguns fatores negativos ou limitações podem ter afetado os resultados obtidos:

Seleção dos estudos relevantes: O conjunto de estudos foi obtido por meio das string de busca apresentada na Seção 2. Foram selecionados todos os estudos existentes sobre Empatia e Mindfulness que apresentavam evidências no desenvolvimento de habilidades socioemocionais baseando-se no conhecimento e experiência dos pesquisadores. Para minimizar tal viés, foram definidos critérios claros de inclusão e exclusão, bem como a definição e delimitação do escopo da pesquisa e uma checagem por mais de um pesquisador oferecido pelo SUMARIZE. Mesmo assim, diferentes pesquisadores podem ter entendimentos diferentes sobre esses critérios, sendo assim, os resultados de seleção de diferentes pesquisadores tendem a variar e devido ao alto número de estudos relacionados a Empatia foi necessária uma diminuição do escopo excluindo estudos secundários estrangeiros e de estudos com participantes de nível superior que não tinham como foco a área/subárea "Socioemocional - Formação de Professores" listada na QPS 14. Além disso, houve a tentativa na utilização de curingas $(*$, ?) para a busca de palavras-chave no plural. Entretanto algumas bases não aceitaram e não retornavam nenhum estudo ao utilizá-las e isso pode ter excluído alguns trabalhos relevantes. 
Externa: O mapeamento foi realizado com limitação temporal, foram catalogados estudos até novembro de 2019. Portanto, os resultados deste estudo podem não englobar todas as evidências já desenvolvidas ou propostas na literatura. Ainda assim, devido ao grande número de documentos analisados, a pesquisa permite generalizar as conclusões acerca do tema.

Extração dos dados: A extração de dados foi realizada por meio das ferramentas de extração presente no SUMARIZE. Nela foram adicionadas as informações relevantes ligadas às questões de pesquisa deste estudo.

Durante todo o processo de extração, foram estabelecidos critérios de como obter os dados dos estudos. Caso surgissem dúvidas, era reservado um tempo para que os pesquisadores pudessem analisar com maior grau de detalhamento e assim concluir os trabalhos.

\section{Considerações Finais}

Este artigo teve como objetivo, de forma sistemática, mapear os estudos do estado da arte visando encontrar evidências para o desenvolvimento de habilidades socioemocionais, tais como Empatia e Mindfulness, aplicáveis às crianças do século XXI integrantes do Ensino Básico brasileiro e, posteriormente, incluídos estudos que tinham como foco o Ensino Superior, servindo como subsídio aos gestores para a criação de políticas públicas a serem aplicados ao ensino básico brasileiro. De um conjunto inicial de 4564 publicações, 96 atenderam aos critérios de inclusão e exclusão, 79 referentes a Empatia e 17 a Mindfulness. A análise dessas publicações possibilitou obter um panorama sobre as tecnologias educacionais digitais/analógicas que vêm sendo utilizadas no contexto do desenvolvimento de habilidades socioemocionais no cenário brasileiro e estrangeiro, como e onde vêm sendo aplicadas, e os resultados obtidos com sua aplicação. Os dados mostram que há, sim, evidências de publicações que desenvolvem habilidades socioemocionais que são aplicáveis a crianças do século XXI no ensino básico brasileiro e que em sua maioria são apoiados por métodos/técnicas estatísticos, revelando um rigor na validação de seus resultados.

Em relação a Empatia, no geral foi observado a concentração dos estudos em um públicoalvo (alunos). Em contexto brasileiro, há uma concentração dos estudos nas regiões sul e sudeste, e levando em conta a nossa população em fase escolar, a quantidade de estudos ainda é muito pequena. Dessa forma fica evidente a necessidade de replicações/novas pesquisas, as quais diversifiquem seu público-alvo, incluindo novos participantes (pais, funcionários, etc.) e em contexto brasileiro uma diversificação das regiões escolhidas.

Em relação a Mindfulness, como lacunas a serem desenvolvidas em estudos futuros podese pontuar, como apresentado por (EM16), a necessidade de replicação dos estudos em ambiente escolar brasileiro, tendo em vista o estado embrionário do Mindfulness na educação do Brasil. Por se tratar de aplicações estrangeiras, faz-se necessário também a adaptação dos protocolos e questionários usados para aferir o desenvolvimento dos alunos. Além disso, o uso/criação de tecnologias que pudessem ajudar na prática de Mindfulness poderia ser muito importante no processo de ensino-aprendizado e a integração de Mindfulness com outras disciplinas pode facilitar sua adoção.

\section{Agradecimento}

O presente capítulo foi realizado com apoio da FAPITEC/SE (Fundação de Apoio à Pesquisa e a Inovação Tecnológica do Estado de Sergipe). 


\section{Referências}

Alfano, B. (2020). Número de alunos no Brasil volta a cair pelo terceiro ano consecutivo. Disponível em: https://oglobo.globo.com/sociedade/educacao/numero-de-alunos-no-brasilvolta-cair-pelo-terceiro-ano-consecutivo-24222590.

Batson, C. D., Lishner, D. A., \& Stocks E. L. (2015). 13 The empathy-altruism hypothesis. In D. A. Schroeder \& W. G. Graziano (Eds.), Oxford library of psychology. The Oxford handbook of prosocial behavior (pp. 259-281). doi: 10.1093/oxfordhb/9780195399813.013.023 [GS_Search]

Bernárdez, B., Durán, A., Parejo, J. A., \& Ruiz--Cortés, A. (2014). A controlled experiment to evaluate the effects of mindfulness in software engineering. In Proceedings of the 8th ACM/IEEE International Symposium on Empirical Software Engineering and Measurement (pp. 1-10). doi: 10.1145/2652524.2652539 [GS Search]

Colagrossi, A. L. R., \& Vassimon, G. (2017). A aprendizagem socioemocional pode transformar a educação infantil no Brasil. Construção psicopedagógica, 25(26): 17-23. Disponível em: $<$ http://pepsic.bvsalud.org/scielo.php?script=sci_arttext\&pid $=\mathrm{S} 1415$ $\underline{69542017000100003 \& \operatorname{lng}=\text { pt\&nrm=iso }>\text { [GS_Search] }}$

Cheng, Y., Chiang, H-C, Ye, J., \& Cheng, L-H (2010). Enhancing empathy instruction using a collaborative virtual learning environment for children with autistic spectrum conditions. Computers \& Education, 55(4): 1449-1458. doi: 10.1016/j.compedu.2010.06.008. [GS_Search]

Cordazzo, S. T. D., \& Vieira, M. L. (2007). A brincadeira e suas implicações nos processos de aprendizagem e de desenvolvimento. Estud. pesqui. psicol., Rio de Janeiro, 7(1). Disponível em: $\quad$ http://pepsic.bvsalud.org/scielo.php?script=sci arttext\&pid=S1808$\underline{42812007000100009 \& \operatorname{lng}=\text { pt\&nrm=iso. }}$ [GS Search]

Cornelius-White, J. (2007). Learner-centered teacher-student relationships are effective: A metaanalysis. Review of educational research 77(1): 113-143. doi: 10.3102/003465430298563. [GS Search]

Davis, D. M., \& Hayes, J. A. (2011). What are the benefits of Mindfulness? A practice review of psychotherapy-related research. Psychotherapy, 48(2): 198. doi: 10.1037/a0022062. [GS Search]

De Waal, F. BM. (2008). Putting the Altruism Back Into Altruism: The Evolution of Empathy. Annual review of psychology, 59, 279-300. Disponível em $\underline{\text { https://www.annualreviews.org/doi/abs/10.1146/annurev.psych.59.103006.093625 [GS }}$ Search]

Erbe, R., \& Lohrmann, D. (2015). Mindfulness Meditation for Adolescent Stress and Well-Being: A Systematic Review of the Literature with Implications for School Health Programs. Health Educator, 47(2), 12-19. Disponível em: https://eric.ed.gov/?id=EJ1153619 [GS Search]

Fadel, C. (2008). 21st Century Skills: How can you prepare students for the new Global Economy?. Disponível em: https:/www.oecd.org/site/educeri21st/40756908.pdf. [GS Search]

Garaigordobil, M. (2012). Evaluation of a program to prevent political violence in the Basque conflict: effects on the capacity of empathy, anger management and the definition of peace. Gaceta sanitaria 26(3): 211-216. doi: 10.1016/j.gaceta.2011.06.014. [GS Search] 
Idoeta, P. A. (2020). Trabalhos dos sonhos de jovens de hoje correm risco de não existir no futuro. Disponível em: https://www.bbc.com/portuguese/internacional51198292?.ocid=wsportuguese.chat-apps.in-app-msg.whatsapp.trial.link1 .auin.

Jones, S. M., \& Doolittle, E. J. (2017). Social and emotional learning: Introducing the issue. The Future of Children. The Future of Children, 27(1), 3-11. doi: 10.2307/44219018. [GS Search]

Kaplan, S. (2001). Meditation, restoration, and the management of mental fatigue. Environment and behavior, 33(4): 480-506. doi: 10.1177/00139160121973106 [GS Search]

KFC. Model UN Teaches the 10 Future Skills Employers Want, 2016. Disponível em: https://bestdelegate.com/model-un-teaches-the-10-future-skills-employers-want/.

Kitchenham, B. A., \& Charters, S. (2007). Guidelines for performing Systematic Literature Reviews in Software Engineering. Keele University and University of Durham, EBSE Technical Report Version 2.3. Disponível em: https://d1wqtxts 1xzle7.cloudfront.net/35830450/2_143465389588742151.pdf?1417739236 $=$ \&response-contentdisposition=inline \%3B +filename\%3DSystematic Literature Reviews SLR.pdf\&Expires=1 607377859\&Signature $=$ JAE1NNcscgAdY $\sim$ eVa8P QVtCMobFoHDhy5f8sNP65YMxMY Gilpx2X4foHxO 8DhDBoPMNcL1u4Ug9gFvIXTCBbmS06Zq3Qdlc8wBcgtyWZobFHNFLsEAZf $\sim$ K1o2Ux1 Tkg0PquOuWeTrv5D6GQEizJMqzew VEd7ebIQ4C57ibt44WGkJIQbVpxcJgTxxWDAOinZheP7J7WNyiLkqUf7wy1QPuYZE4n 7fuWr23h7HV touJEh-UoFXre1tdRe0Wp-0c1VhwWYQimQ-WTG3XEtXotXJUcHOyThCtQNxN6-4r-YNasVj gSbGu9rBZcTRhabyaEtrjU8EnbRcPN5MA_\&Key-Pair$\underline{\mathrm{Id}=\mathrm{APKAJLOHF} 5 \mathrm{GGSLRBV4ZA} \text { [GS Search] }}$

Long, Edgar CJ, Jeffrey J., A., \& Mikiyasu, H. (2008). Transferable principles from a formative evaluation of a couples' empathy program. Journal of Couple \& Relationship Therapy 7(2): 88-112. doi: 10.1080/15332690802106828. [GS Search]

Madaliyeva, Z., Mynbayeva, A., Sadvakassova, Z., \& Zholdassova, M. (2015). Correction of Burnout in Teachers. Procedia-Social and Behavioral Sciences 171: 1345-1352. doi: 10.1016/j.sbspro.2015.01.252 [GS Search]

Ministério da Educação (MEC). Base Nacional Comum, 2019. Página Inicial. Disponível em: http://basenacionalcomum.mec.gov.br/.

Oliveira, E. (2018). Quase 4 em cada 10 jovens de 19 anos não concluíram o ensino médio, aponta levantamento. Disponível em: https:/g1.globo.com/educacao/noticia/2018/12/18/quase-4em-cada-10-jovens-de-19-anos-nao-concluiram-o-ensino-medio-apontalevantamento.ghtml.

ONU. Sustainable Development, 2015. Disponível em: https://sustainabledevelopment.un.org/post2015/transformingourworld.

Paranhos, M. (2016). Relações entre habilidades socioemocionais e inovação para alguns licenciandos em ciências biológicas. Universidade Federal de Sergipe, p. 27-34,2016. Disponível em: https://core.ac.uk/download/pdf/197138767.pdf [GS Search]

Pensar Contemporâneo. (2019). A Empatia é ensinada a estudantes de 6 a 16 anos nas escolas da Dinamarca. Disponível em: https://www.pensarcontemporaneo.com/a-empatia-e-ensinada-aestudantes-de-6-a-16-anos-nas-escolas-dadinamarca/?fbclid=IwAR0UlIuCAu9iE0iK4zqkTZN1stdgYP372e4szVngi4bqx_cqmhvfrd9 DY . 
Petersen, K., Feldt, R., Mujtaba, S., \& Mattsson, M. (2008). Systematic Mapping Studies in Software Engineering. Proceedings of the 12th International Conference on Evaluation and Assessment in Software Engineering. 17. doi: 10.14236/ewic/EASE2008.8 [GS Search]

Ponsoda, F. C. (2017). The effect of an out-of-school Mindfulness program on adolescents' stress reduction and emotional wellbeing. Cuadernos de Psicología del Deporte, 17(3), 35-44. Disponível em https://psycnet.apa.org/record/2018-30927-003 [GS Search]

Ruic, G. (2019). Estes são os países mais felizes do mundo. Disponível em: https://exame.com/mundo/estes-sao-os-paises-mais-felizes-do-mundo-em-2019/

Santos, C. G., Nunes M. A. S. N., \& Romero, M. (2019). Guia de atividades desplugadas para o desenvolvimento do pensamento computacional. [] Porto Alegre, SBC, 201940 p.: il (Almanaque para a popularização de ciência da computação. Série 12, Guia pedagógico; v2) ISBN 978-85-7669-474-8. Disponível em: http://almanaquesdacomputacao.com.br/gutanunes/publications/serie12/S12V2small.pdf.

Senado Federal. (1996). Lei de Diretrizes e Bases da Educação Nacional. Disponível em: https://www2.senado.leg.br/bdsf/bitstream/handle/id/70320/65.pdf .

Swan, P., \& Riley, P. (2012). Mentalization: A tool to measure teacher empathy in primary school teachers. Australian Association for Research in Education/APERA Conference, Sydney. Disponível em https://eric.ed.gov/?id=ED544520 [GS Search]

UNESCO. Global Citizenship Education: Topics and Learning Objectives, 2016. Disponível em: https://www.gcedclearinghouse.org/sites/default/files/resources/150020por.pdf. [GS_Search]

Sahin, M. (2012). An investigation into the efficiency of empathy training program on preventing bullying in primary schools. Children and Youth Services Review 34(7): 1325-1330. doi: 10.1016/j.childyouth.2012.03.013 [GS Search]

Silva, I. D., Nunes, M. A. S. N. , Felizardo, K. E., Nakagawa, E. Y., Ferrari, F. C., Fabbri, S. C. P. F., \& Júnior, J. H. S. (2018). Almanaque Para Popularização De Ciência Da Computação Série 6: Metodologia Científica e Tecnológica; Volume 7: Mapeamento Sistemático PARTE 1. 1. ed. Porto Alegre: SBC, 2018. v. 7. 36p. Disponível em: http://almanaquesdacomputacao.com.br/gutanunes/publications/serie6/S6V7small.pdf.

Warren, C., \& Lessner, S. (2014). "Who has Family Business?" Exploring the Role of Empathy in Student-Teacher Interactions. Perspectives in Urban Education. 11: 122-131. Disponível em https://eric.ed.gov/?id=EJ1044080 [GS Search] 